Research Article

\title{
Deformation Response and Safety Evaluation of Buildings Affected by Subway-Station Construction
}

\author{
Chunlei Feng $\left(\mathbb{D},{ }^{1}\right.$ Dingli Zhang, ${ }^{2}$ Hualao Wang, ${ }^{1}$ and Xuan Zhang ${ }^{1}$ \\ ${ }^{1}$ Bridge and Tunnel Research Center, Research Institute of Highway, Ministry of Transport, Beijing 100088, China \\ ${ }^{2}$ Key Laboratory for Urban Underground Engineering of Ministry of Education, Beijing Jiaotong University, \\ Beijing 100044, China \\ Correspondence should be addressed to Chunlei Feng; fclbjtu@163.com
}

Received 22 April 2021; Accepted 24 May 2021; Published 8 June 2021

Academic Editor: Faming Huang

Copyright (c) 2021 Chunlei Feng et al. This is an open access article distributed under the Creative Commons Attribution License, which permits unrestricted use, distribution, and reproduction in any medium, provided the original work is properly cited.

Based on the sand and pebble stratum in the Beijing area, this paper studies the interaction between deep-foundation-pit excavation for subway stations and surrounding buildings using an orthogonal test. Moreover, it considers the relative position relationship between buildings and how the foundation pit is set up as well as different design schemes for foundation pits and the surrounding buildings. Results show that the horizontal distance $s$ between the building and foundation pit and stiffness of the building itself have a clear impact on the differential settlement $\delta_{i j}$ and relative deflection $\Delta$, and the degree of deformation of the building near the corner of the foundation pit is complex. Simultaneously, based on numerical simulation results, the deformation characteristics and degree of deformation of the building under different relative position relationships with the foundation pit are analyzed. Finally, by establishing a relationship among the comprehensive deformation index $D_{j}$, surrounding environmental safety evaluation index $S_{j}$, and scheme safety grade $L_{j}$, a multiangle safety evaluation method for buildings affected by foundationpit construction is formed, which can provide a reference for the research and design of similar projects.

\section{Introduction}

Deep-foundation-pit engineering is accompanied by different degrees of environmental impact in construction processes, which causes a change in the stress field of surrounding soil, resulting in foundation soil deformation within a certain range that affects surrounding buildings and structures. If foundation-pit excavation deformation is not properly controlled, it can threaten the safety of the surrounding environment because the deformation of the surrounding soil leads to uneven settlement or even damage caused by cracking in the building and structure, and the building can then no longer be used for its normal purpose. However, damage to or the destruction of the surrounding buildings also has an impact on deep-foundation-pit engineering. For example, the excessive settlement of the adjacent buildings exacerbates the deformation of the surrounding soil, which may lead to a further increase in the deformation of the retaining structure. Moreover, cracks and leaks in underground pipelines may cause water bubbles at the bottom of the foundation pit and threaten the stability of its basement [1]. Therefore, with the increasing depth of foundation-pit excavation and increasingly complex surrounding environment, the foundation-pit support system should not only be appropriate for its own strength and stability but should also meet the requirements of the surrounding environment for deformation control.

At present, existing research on the environmental response of deep-foundation-pit excavation mostly focuses on the influence of foundation-pit deformation on surrounding structures, including actual deformation statistics [2-4] and numerical simulations and model test [5-7]. Li and Yan [8] numerically simulated ground surface settlement using auxiliary planes perpendicular and parallel to the excavation and angled auxiliary planes at the excavation corner; thereafter, they analyzed the bending and torsional deformation regularities of the surrounding buildings and developed an estimation method for building settlement. Liang 
et al. [9] investigated building settlement near excavations in soft clay and proposed a simplified theoretical method to predict the additional settlements and axial forces of excavation adjacent to existing building floating piles in soft clay. Combined with the deep-foundation-pit engineering for subway stations in their respective regions, many scholars [10-13] have conducted in-depth research on deformation characteristics and influencing factors. Moreover, many studies $[14,15]$ have reported the response of surrounding buildings to foundation-pit excavation using field measurements, which provides a basis for studying the deformation mode and allowable deformation of buildings. According to the research of Skempton and MacDonald [16] and later scholars, damage to buildings can be roughly divided into architectural, functional, and structural damages. On the basis of previous research, Burland [17] proposed a classification standard for the levels of damage to buildings according to the degree of repairability of the largest cracks in masonry walls. To clearly and accurately quantify the impact of foundation-pit excavation on the surrounding environment, Burland and Wroth [18] provided a definition for various building-deformation variables, which has been widely recognized by related research.

Through a broad review of the relevant literature, it can be established that existing studies have the following limitations: first, foundation-pit engineering and the surrounding environment are an interactive system; therefore, accurately analyzing the interaction relationship between them depending only on measured analyses and theoretical research is difficult; second, a finite-element method can establish a variety of working conditions, and the influence of different factors on results can be further analyzed using calculation results. However, existing studies have only established the relative displacement relationship between the surrounding buildings and foundation pit. Because foundation pits for subway stations are long and narrow, the deformation of a building at a particular location can hardly represent the entirety of the deformation around the foundation pit; third, in the research process, the influence of the foundation pit itself and the surrounding environment on the deformation results has not been fully considered, and the calculation results have not been further combined with multiple factors to form a specific impact evaluation.

In this study, a sandy pebble stratum formed the basis of the investigation, and related engineering data were used to establish stratigraphic distribution and a standardized model for a subway-station deep-foundation pit. Starting from the influence of foundation-pit excavation on building deformation, the interaction between foundation-pit excavation and adjacent buildings is analyzed and evaluated in many aspects, so as to give feedback about the influence of foundation-pit excavation on the environment. Secondly, starting from the design scheme of the foundation-pit support system and the relative displacement relationship between the building and the foundation pit, the distance between the building and the foundation pit, the stiffness of the building itself, the stiffness of the retaining structure of the foundation pit, and the stiffness of the inner support are set as the main influencing factors. An orthogonal test and research into the deformation of the different test schemes were then used to analyze the degree of influence of the various factors on building deformation. A comprehensive evaluation method for the influence of multiangle foundation-pit excavations on surrounding buildings was then developed, which provides a reference for subsequent related research and design.

\section{Project Overview}

2.1. Project Introduction. The project is located in Beijing, China, and its geographical location is shown in Figure 1. As the political and cultural center of China with a long history, Beijing has a varied construction style, which is complex and spans a large area; thus, given the complex urban environmental conditions, the requirements for environmental protection in relation to foundation-pit engineering are high, especially with regard to foundation-pit engineering for subway stations. As a form of urban construction, these foundation pits are excavated in considerably populated areas and can therefore cause a serious engineering accident if sufficient care is not taken. In this study, foundation-pit engineering for the first and second phase of the subwaystation construction of Beijing Metro Line 6 is taken as the focus, and 16 open-cut foundation-pit projects are selected as the main research objects. The support system adopts the form of "internal support + bored pile." The specific dimensions and characteristic values of the foundation-pit excavation are shown in Table 1 , where, $L, B, H_{\mathrm{e}}$, and $H$ are the length, width, excavation depth, and depth of the foundation pit's retaining structure. The diagram of subway lines is shown in Figure 2 in which the pink line is the first phase of the project, and the green line is the second. The serial numbers of each station in the figure correspond to the serial numbers in Table 1.

2.2. Geological and Hydrological Conditions of the Project. The first and second phases of Beijing Metro Line 6 are located in central and eastern Beijing, where the soil is mainly cohesive, silty, sandy, and with pebbles. Based on the summary of the geological survey data for the Beijing Metro Line 6 project, the stratigraphic sequence of the whole area from top to bottom is shown in Table 2, and the main types of soil in each layer are shown in Figure 3.

The Line 6 subway stations are mainly located at a depth of 15-25 $\mathrm{m}$ underground, and they are all built in the Quaternary clay, sand, and pebble layer. Therefore, the influence of strata conditions on subway construction in Beijing mainly includes the following two aspects [19]:

(1) The sand and pebble stratum in Beijing are a typical mechanical unstable stratum, which is characterized by loose structure, lack of cementation, and different sized granulation. Once this kind of stratum is excavated, the original state of relative stability or equilibrium is easily destroyed so that the excavation face and wall of the foundation pit lose their constraints, leading to instability. Large pores are present among the grains of the sandy pebble 


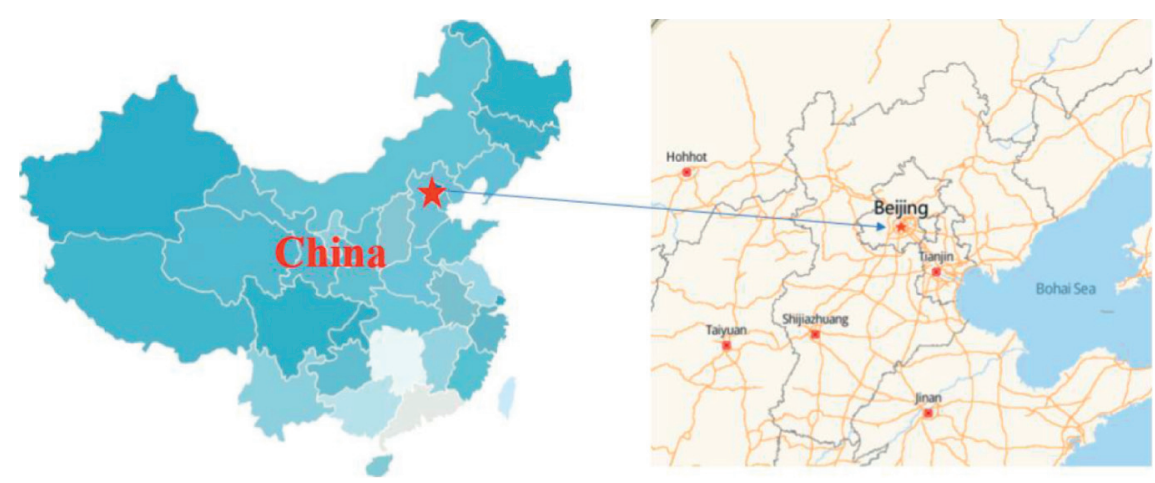

FIGURE 1: Schematic of the project location.

Table 1: Basic statistics of the excavation for Beijing Metro Line 6 open-cut station.

\begin{tabular}{|c|c|c|c|c|}
\hline Project number & $L(\mathrm{~m})$ & $B(\mathrm{~m})$ & $H_{\mathrm{e}}(\mathrm{m})$ & $H(\mathrm{~m})$ \\
\hline 1 & 381 & 34.2 & 24.4 & 27.7 \\
\hline 2 & 245.3 & 22.3 & 28.7 & 34.9 \\
\hline 3 & 291.1 & 28.8 & 18.5 & 30.7 \\
\hline 4 & 323.8 & 23 & 17.4 & 30.4 \\
\hline 5 & 219.1 & 20.9 & 19.8 & 23.3 \\
\hline 6 & 564.9 & 19.7 & 21.1 & 25.4 \\
\hline 7 & 358.3 & 21.1 & 17.5 & 22.5 \\
\hline 8 & 256 & 19.5 & 18.5 & 21 \\
\hline 9 & 394 & 34.9 & 18.6 & 21 \\
\hline 10 & 357.5 & 20.9 & 13.3 & 17.8 \\
\hline 11 & 473 & 43.9 & 22.2 & 26.6 \\
\hline 12 & 219.6 & 21.9 & 17.6 & 33.6 \\
\hline 13 & 314.2 & 21.1 & 17.9 & 42.5 \\
\hline 14 & 326.4 & 20.9 & 20 & 24.2 \\
\hline 15 & 286.4 & 20.8 & 17.4 & 22.4 \\
\hline 16 & 523.6 & 29.7 & 18.2 & 22.4 \\
\hline Average & 319.5 & 25.44 & 20.12 & 27.16 \\
\hline
\end{tabular}

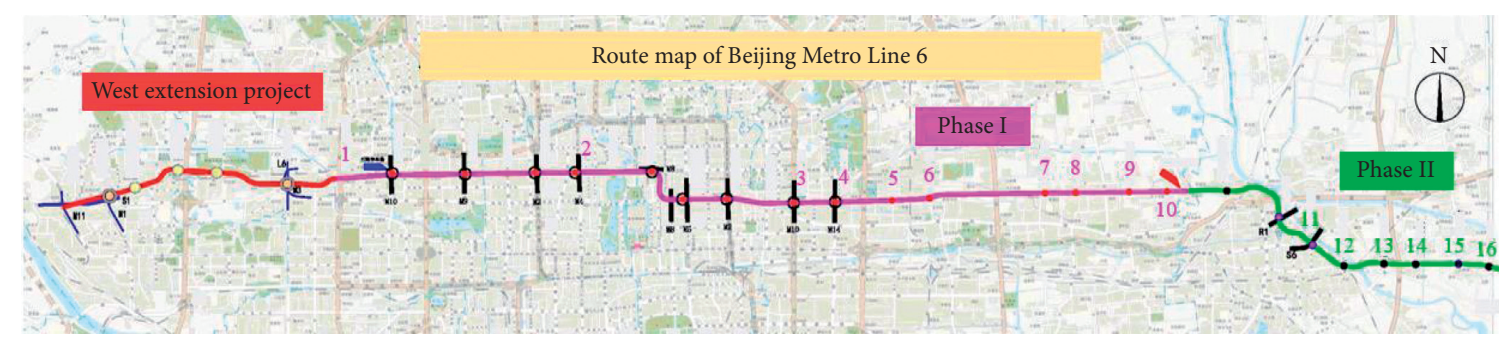

Figure 2: Diagram of Beijing Metro Line 6 and project numbers.

TAвLE 2: Strata along Beijing Metro Line 6.

\begin{tabular}{|c|c|c|}
\hline Number & Name of the soil & Type of soil \\
\hline 1 & Artificial filled soil & $\begin{array}{l}\text { It mainly contains silt filling soil and miscellaneous filling soil, in which the } \\
\text { miscellaneous filling soil is } 6-8 \mathrm{~m} \text { below the ground }\end{array}$ \\
\hline 2 & Recently deposited soil & It mainly contains silty soil layer, silty clay layer, and fine sand \\
\hline 3 & $\begin{array}{l}\text { Quaternary Holocene alluvial and } \\
\text { diluvial deposits }\end{array}$ & It mainly contains silty soil, silty clay, clay, fine silty sand, and medium coarse sand \\
\hline 4 & $\begin{array}{l}\text { Quaternary late Pleistocene alluvial and } \\
\text { diluvial deposits }\end{array}$ & $\begin{array}{l}\text { It mainly contains boulder, medium coarse sand, fine silty sand, silty clay, clay, silt, and } \\
\text { fine medium sand }\end{array}$ \\
\hline
\end{tabular}




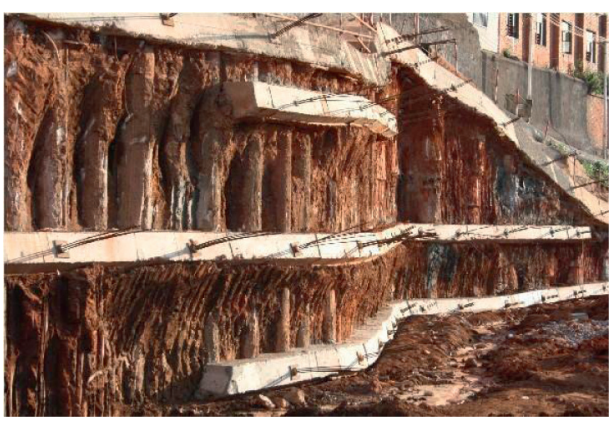

(a)

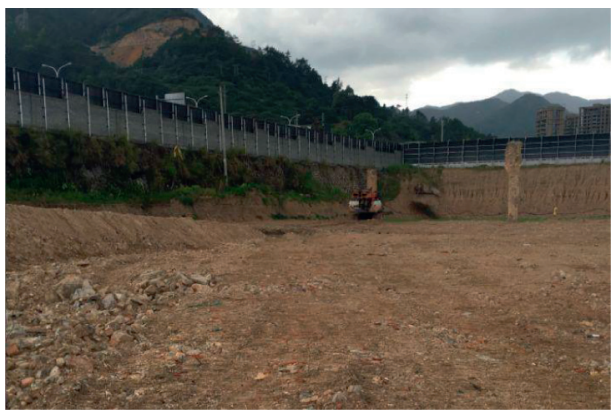

(c)

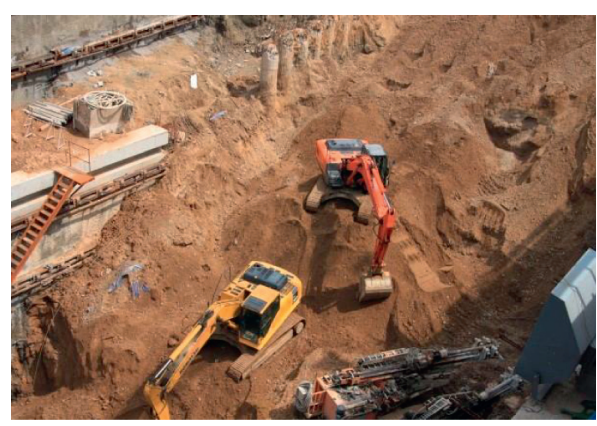

(b)

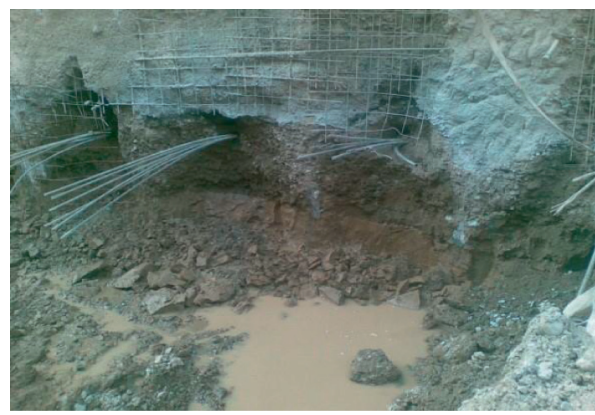

(d)

Figure 3: Longitudinal geological profile of the K0 $+600-\mathrm{K} 1+074$ section of the Gongchang road project. (a) Silty clay. (b) Fine powder sand. (c) Sandy pebble. (d) Sandy pebbles are interlaced with silty clay.

formation, and the cohesive force among the grains is almost zero. During the excavation of the foundation pit, the stratum can easily collapse. In particular, the spalling of large pebbles in the wall of the foundation pit may cause a sudden subsidence of the overlying strata.

(2) Beijing strata also contain a large number of sand layers, which are generally dense but have poor selfstabilizing ability, and these layers are prone to collapse. In the presence of water, sand flow occurs easily. If precipitation construction is adopted, the surrounding groundwater level decreases, and the silty sand layer becomes loose as a result of water loss, which makes it unstable when disturbed by an excavation operation.

Therefore, studying the influence of subway-station construction on the surrounding buildings in dense urban areas with these strata characteristics is necessary to establish a set of construction safety evaluation methods.

\section{Basic Information on the Foundation Pits and Surrounding Buildings}

A large number of engineering design data pieces were collected to standardize the design parameters of the subway-station foundation pits in the Beijing area, and the finite-element calculation method was adopted to analyze the interaction between the existing design scheme for foundation-pit excavation and the surrounding buildings.
3.1. The Establishment of a Foundation-Pit Standard Model. After assigning an average weighting to all the parameters of the open-cut foundation pit, as shown in Table 1, the main parameters of the standardized model of the subway-station foundation pit were obtained (Table 3), where $d, l, H$, and $E$ are the diameter, spacing, depth, and stiffness, respectively, of the bored piles. The excavation conditions of the standardized foundation pit are shown in Table 4.

3.2. Main Building Parameters and Relative Position Relationship. The overall stiffness of most multistory masonry structures, compared to reinforced concrete shear frame structure, is weaker due to its shallow depth and overall stiffness, making it relatively more vulnerable to environmental impact in the excavation of foundation pits. Therefore, this paper focuses primarily on the deformation of multistory masonry structures to evaluate the environmental impact of foundation-pit excavations.

To study the influence of foundation-pit excavation on surrounding buildings, the setting parameters mainly include the position relationship between the building and envelope structure and the size and stiffness of the building itself. The size of the building is shown in Figure 4. The outer contour is a strip $27 \mathrm{~m}$ long and $4.5 \mathrm{~m}$ wide. Four floors are present, and each layer is $3 \mathrm{~m}$ high, with a total height of $12 \mathrm{~m}$. The main structure of the building includes a horizontal wall, vertical wall, partition wall, and floor slab. The foundation is in the form of a strip foundation under the wall, and the openings for doors and windows have been considered. The thickness of the wall and floor slab is $0.24 \mathrm{~m}$ 
TABLE 3: Main parameters of the normalized model of the Beijing Metro Line 6 open-cut foundation pit.

\begin{tabular}{|c|c|c|}
\hline \multirow{3}{*}{ Basic dimensions } & $L(\mathrm{~m})$ & 319.5 \\
\hline & $B(\mathrm{~m})$ & 25.44 \\
\hline & $H_{\mathrm{e}}(\mathrm{m})$ & 20.12 \\
\hline \multirow{3}{*}{ Bored pile } & $d(\mathrm{~m})$ & $0.8 / 1.5$ \\
\hline & $l(\mathrm{~m})$ & (1.0/ \\
\hline & $\begin{array}{c}H(\mathrm{~m}) \\
E(\mathrm{MPa})\end{array}$ & $3.0 \times 10^{4}$ \\
\hline \multirow{4}{*}{ Inner support } & Material & Q235 \\
\hline & Layer number & 4 \\
\hline & Section size $(\mathrm{mm})$ & $\Phi 609 \times 14 \Phi 609 \times 16$ \\
\hline & Horizontal spacing $(\mathrm{m})$ & 3 \\
\hline
\end{tabular}

TABLE 4: Main excavated conditions of the foundation pit.

\begin{tabular}{lcc}
\hline Construction step & Construction content & Excavation depth \\
\hline I & Initial ground stress & - \\
II & Retaining structure construction & - \\
III & Excavate the first layer of soil and set up the first steel support & $2 \mathrm{~m}$ \\
IV & Excavate the second layer of soil and set up the second steel support & $4.5 \mathrm{~m}$ \\
V & Excavate the third layer of soil and set up the third steel support & $4.5 \mathrm{~m}$ \\
VI & Excavate the fourth layer of soil and set up the fourth steel support & $4.5 \mathrm{~m}$ \\
VII & Excavation to the bottom & $4.5 \mathrm{~m}$ \\
\hline
\end{tabular}

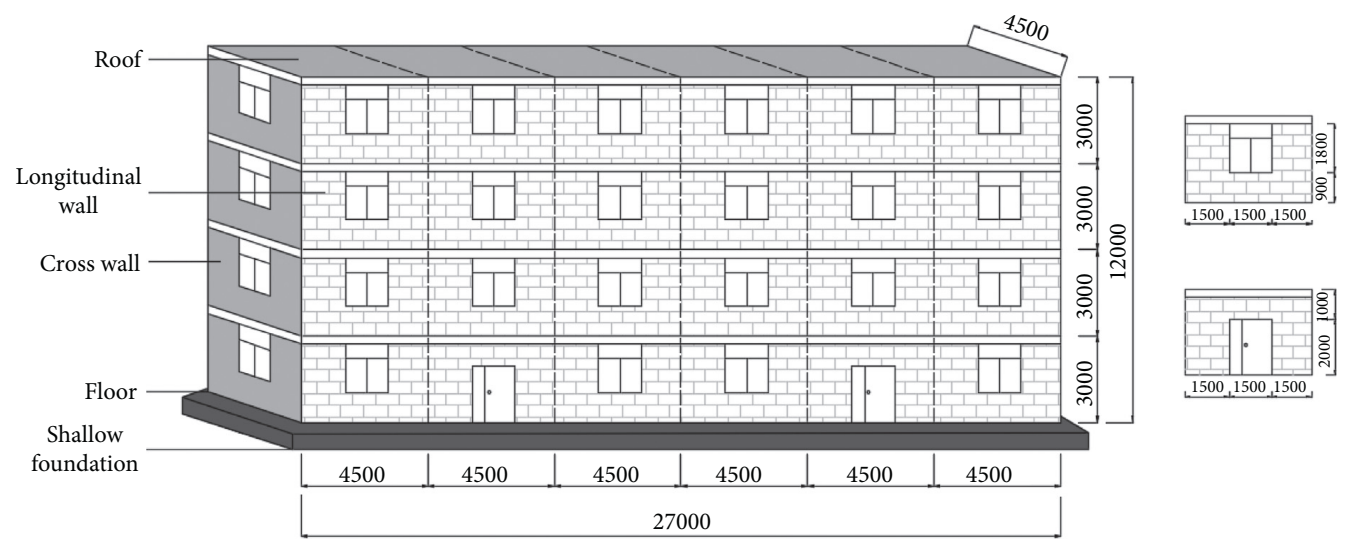

FIGURE 4: Dimensions of surrounding buildings.

and $0.1 \mathrm{~m}$, respectively. Considering that the stiffness of most masonry structures gradually degrades with age, the impact of earthquakes, and other factors [20, 21], the elastic modulus of the wall is set at $220 \mathrm{MPa}$, and Poisson's ratio is 0.1 . The elastic modulus of the floor is set at $30 \mathrm{GPa}$, and Poisson's ratio is 0.2 .

Since the subway-station foundation pit is long and narrow, the deformation in the length direction of the foundation pit is more complicated $[22,23]$. The location of the maximum deformation of the foundation-pit excavation was determined using the design, construction scheme, and complexity of the surrounding environment, of which the influencing factors relating to the deformation of the surrounding buildings include the structural form, buried depth of the foundation, and relative position relationship with the foundation pit.
Therefore, the deformation situation between the building and the foundation pit at different relative positions is compared and analyzed by using three different relative position relations: on one side of the foundation pit near the center, at a quarter of the length, and at an angle to the pit, as shown in Figure 5. The comparative analysis in relation to the different relative positions between the buildings and foundation pit depending on the location parameter is given in the subsequent calculations.

When setting the horizontal distance between the building and envelope, ensuring that the building was located in the main impact area of the foundation-pit excavation was necessary, as shown in Figure 6 . When the longitudinal wall of the building is parallel to the foundation pit, the horizontal wall crosses the position where the maximum settlement occurs on the surface. When the 


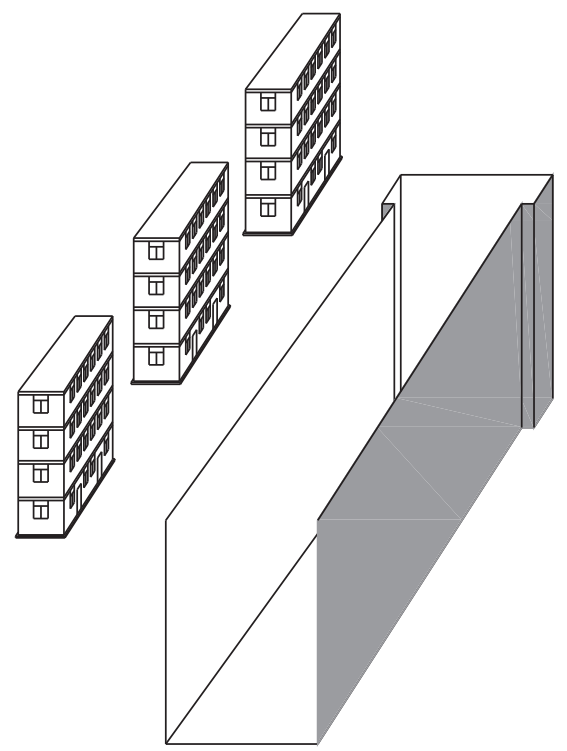

(a)

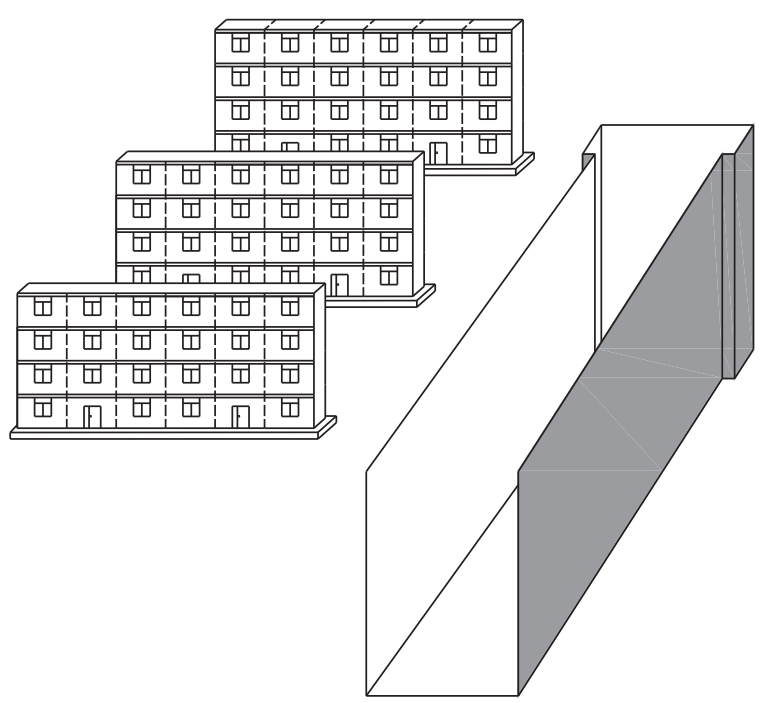

(b)

FIGURE 5: Schematic of the relative position relationship between the building and foundation pit. (a) The longitudinal walls of the building are parallel to the foundation pit. (b) The longitudinal walls of the building are perpendicular to the foundation pit.

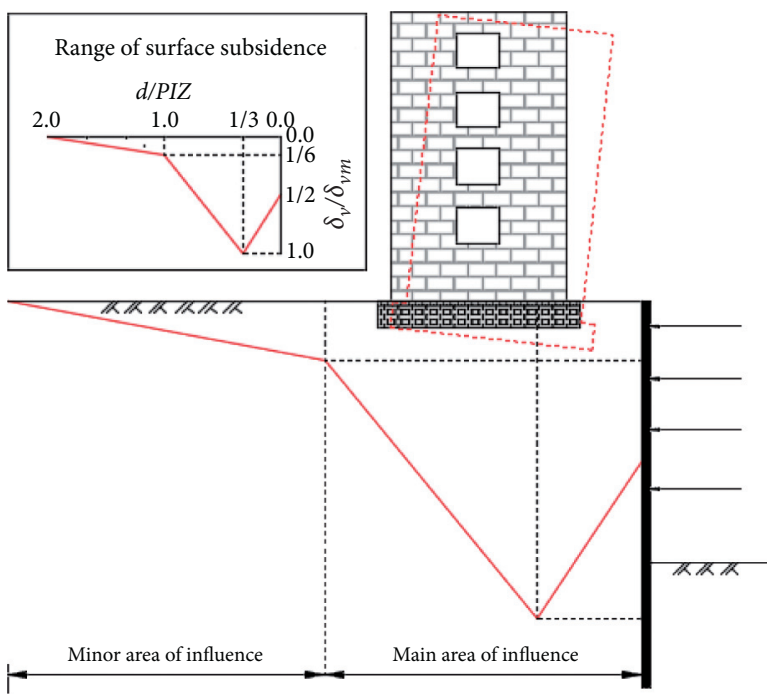

(a)

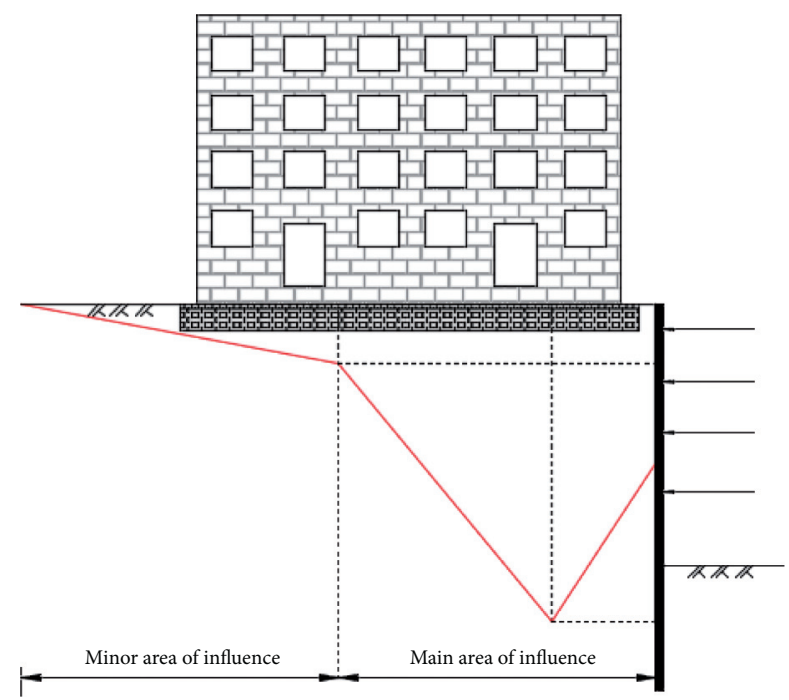

(b)

FIgURE 6: The relationship between the location of the building and influence range of ground subsidence during excavation [24]. (a) The longitudinal walls of the building are parallel to the foundation pit. (b) The longitudinal walls of the building are perpendicular to the foundation pit.

longitudinal wall of the building is perpendicular to the foundation pit, the longitudinal wall crosses the position where the maximum settlement occurs.

3.3. Selection of a Constitutive Model. To describe the stress-strain relationship of soil accurately, scholars have proposed various models to consider the small-strain stiffness of soil. These models can distinguish the deformation characteristics of soil in relation to loading and unloading. Among them, the hardening soil small-strain model proposed by Benz [5], used with small-strain stiffness, has been widely used and recognized by scholars in the field of foundation-pit engineering. Therefore, this paper adopted this constitutive model to simulate and analyze the excavation process of a standardized foundation pit in the Beijing strata. Moreover, considering the calculation efficiency of the finite-element software and symmetry of the foundation 
pit, a half-section of the central axis along the long side of the foundation pit was used for the simulation in the numerical calculation.

\section{Deformation of the Retaining Structure and Surrounding Buildings after Excavation of a Foundation Pit}

To facilitate the description of the deformation under different relative position relationships between buildings and foundation pits, the foundation pits and buildings are numbered using the plane coordinate system as viewed from above. Starting from the pit corner, the order of buildings is stipulated in the direction of the $x$-axis, as shown in Figure 7. In the calculation process, the deformation in the floor and roof of the building was monitored. The deformation results were summarized by the deformation at the intersections of each partition wall with the baseplate and roof. The positions and numbers of the monitoring points are shown in Figure 8: a monitoring point on the roof is $m$, and the number of the monitoring point on the bottom plate perpendicular to the roof is $m^{\prime}$.

When the longitudinal wall of the building is parallel to the foundation pit, as shown in Figure 9, based on the size of the foundation pit, the distance between two adjacent buildings is set as $30 \mathrm{~m}$, and the horizontal distance from the foundation pit is $5 \mathrm{~m}$. Because the width of the foundationpit shield shaft is larger than the shield interwell section, the distance between building $\mathrm{A}$ and the foundation pit is closer than that between building $\mathrm{B}$ and building $\mathrm{C}$, and the horizontal distance is approximately $3 \mathrm{~m}$.

The calculation results are shown in Figure 10. By comparing the soil deformation on the left and right sides of the foundation pit in Figures 10(a) and 10(b), it can be seen that the size and range of horizontal and vertical deformation of the soil with a building on one side of the foundation pit are significantly larger than that without a building. At the same time, further analysis can obtain the deformation of the building, as shown in Figures 11 and 12.

By comparing the floor settlement of the three buildings in Figure 11, it can be seen that: (1) building A, which is closer to the shield shaft, has the largest settlement, and its maximum settlement is approximately $-32.5 \mathrm{~mm}$; the settlement of buildings $\mathrm{B}$ and $\mathrm{C}$ is small, and the maximum settlement is approximately $-25.9 \mathrm{~mm}$. The settlement of buildings $B$ and $C$ is in the form of synchronous subsidence and symmetrical depression on both sides of the floor, and the settlement of building $\mathrm{A}$ is due to the settlement on the left side of the floor being greater than the asymmetric depression on the right side; (2) the length of the red line on the right side of the deformation curve in Figure 11 is the differential settlement of buildings $\mathrm{A}, \mathrm{B}$, and $\mathrm{C}$, which is 24.8 , 18.4, and $25 \mathrm{~mm}$, respectively. The relative deflections are $12.55,9.8$, and $14.35 \mathrm{~mm}$, respectively. The corresponding deflection ratios are $1 / 2151,1 / 2755$, and $1 / 1881$, respectively; (3) the settlement of the front edge of the floor of the three buildings is greater than that of the back edge, and the settlement difference between the front and back sides of the floor is between 5 and $7.5 \mathrm{~mm}$. Based on the previous assumptions on building deformation, it is speculated that the whole building may be inclined toward the foundation pit.

Figure 12 shows the horizontal deformation of the top and bottom monitoring points of the longitudinal wall in front of the building in the direction of the foundation pit. The maximum horizontal displacement of the top and bottom of building $\mathrm{A}$ is 7.5 and $5.5 \mathrm{~mm}$, respectively. The maximum horizontal displacements at the top and bottom of the longitudinal walls of buildings $\mathrm{B}$ and $\mathrm{C}$ are similar, approximately 5.45 and $3.85 \mathrm{~mm}$, respectively. Compared with buildings $\mathrm{B}$ and $\mathrm{C}$, the maximum horizontal displacement of building $\mathrm{A}$ in the direction of the foundation pit appears on the left side of the building, i.e., the side near the shield shaft, and the bottom presents a slightly concave deformation. However, the deformation of buildings B and C is generally shown as an overall forward tilt, without prominent deformation in any position. Therefore, the forms of deformation of the buildings located near shield shafts are relatively complex compared with those in other locations.

4.1. Deformation of Buildings with Longitudinal Walls Perpendicular to the Foundation Pit. As shown in Figure 13, when the longitudinal wall of the building is perpendicular to the foundation pit, with the three buildings still in a horizontal position, the horizontal spacing between the adjacent buildings is $65 \mathrm{~m}$, the horizontal distance between the transverse walls of the building near the foundation pit is $5 \mathrm{~m}$, and horizontal distance between building $\mathrm{A}$ and the foundation pit is $3 \mathrm{~m}$ due to its proximity to where the shield shaft and shield interwell section connect.

As can be seen from Figure 14, when the longitudinal wall of the building is perpendicular to the foundation pit, the deformation range of horizontal and vertical soil displacement on the side of the foundation pit with the building increases compared with that on the side without the building. The maximum horizontal displacement is within the range of $30-40 \mathrm{~mm}$, and the maximum vertical settlement is within the range of $40-50 \mathrm{~mm}$.

When the longitudinal wall of the building is perpendicular to the foundation pit, the horizontal displacement of the building toward the foundation pit can be ignored because the moment of inertia along the vertical wall of the building is much larger than that along the horizontal wall. As shown in Figure 15, through further analysis of the settlement deformation at the bottom of the building, it can be seen that the left and right sides of the floor of the building at the three locations show synchronous settlement deformation, and the maximum settlement is similar at approximately $30 \mathrm{~mm}$. The three largest settlement points are all located near monitoring points no. 6 (or no. 12) and no. 7 (or no. 13). This means that the horizontal distance from the ipsilateral retaining structure of the foundation pit is approximately $0.7-0.8 H_{\mathrm{e}}$ within the range of $12-14 \mathrm{~m}$.

In summary, when the longitudinal wall of the building is perpendicular to the foundation pit, the horizontal displacement of the building toward the foundation pit is 


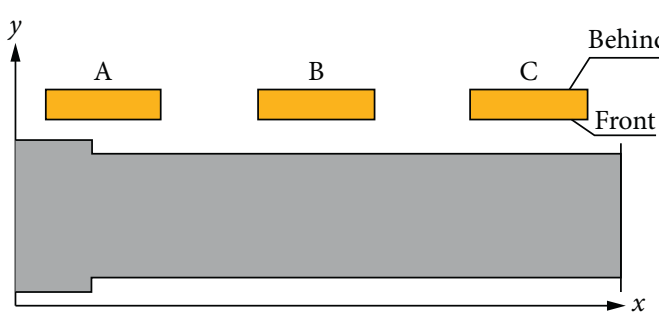

(a)

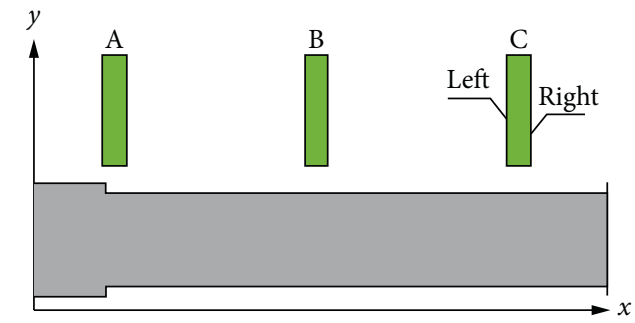

(b)

FIgURE 7: The position relationship between the foundation pit and building, as viewed from above, in plane coordinate and building number.

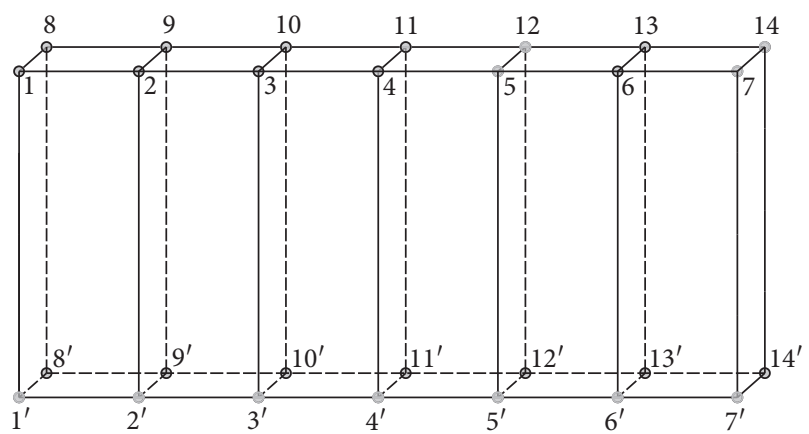

Figure 8: Diagram of building-deformation monitoring points.

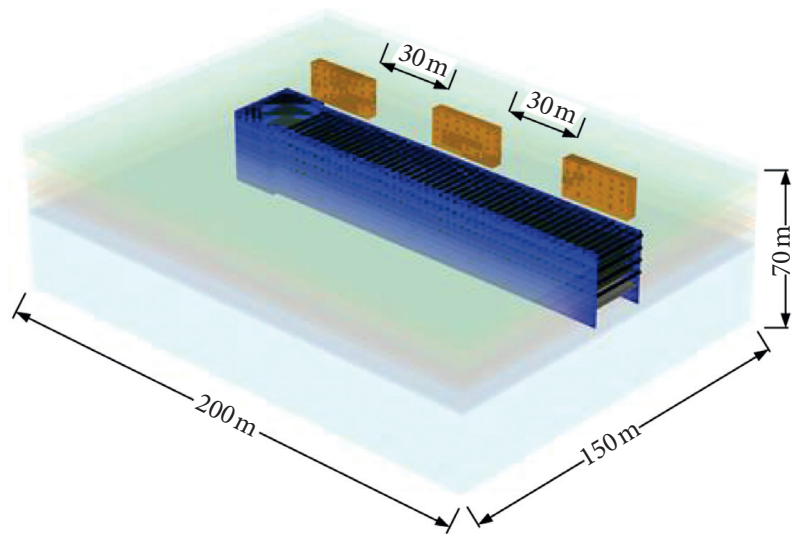

Figure 9: Three-dimensional numerical model of the longitudinal wall of buildings parallel to the foundation pit.

ignored due to the greater stiffness of the vertical wall. Therefore, the settlement deformation of the floor of the building is mainly analyzed herein. It can be seen from the building settlement deformation curve that the position of the maximum settlement of the building is consistent with the position of the maximum settlement of the surface outside the pit obtained from the measured data statistics [18]. Therefore, further studies on the influence of various factors on building deformation can be conducted in subsequent studies based on the above 3D model.

\section{Environmental Response and Safety Evaluation of Foundation-Pit Construction}

To obtain the degree of influence of the excavation of the foundation pit on the surrounding buildings accurately, this section combines the main parameters of the buildings and foundation-pit support scheme and analyzes the influence of different support schemes and the state of the surrounding buildings on the deformation of the building in the process of foundation-pit excavation from multiple angles.

According to the deformation described in the previous section, the forms of deformation in the buildings are primarily uneven settlement $\delta_{i j}$, overall deflection $\Delta$, inclination toward the pit (angle $\alpha$ ), and torsion (angle $\beta$ ), as shown in Figure 16. Uneven settlement $\delta_{i j}$ in Figure 16(a) is the absolute value of the difference between the settlement value of the monitoring point $j$ with the largest settlement and that of the monitoring point $i$ with the smallest settlement of the monitoring points on the floor.

5.1. Deformation Response Analysis of the Surrounding Buildings Affected by Foundation-Pit Construction. In this section, the foundation-pit supporting scheme is represented by two main factors $E^{e}$ and $E^{s}$, while the relative position relationship between the building and the foundation pit, $s$ and $E^{c}$, is mainly considered for the surrounding buildings, as shown in Table 5, where $s$ is the horizontal distance between the building and foundation pit; $E^{c}$ is the stiffness of the building wall; $E^{e}$ is the stiffness of the retaining structure; and $E^{s}$ is the stiffness of the internal support. Three levels are set for each factor. The relative position relationship between the building and foundation pit is consistent with that in Section 4.

Through the analysis and study of different foundationpit retaining design schemes and the deformation of the surrounding buildings in the previous section, the specific degree of influence of the different factors on the deformation of buildings with the three relative positions can be obtained, as shown in Figure 17. The vertical coordinate in the figure is the absolute value of the difference between the maximum and minimum mean values of the deformation parameters obtained at the three different levels under the 


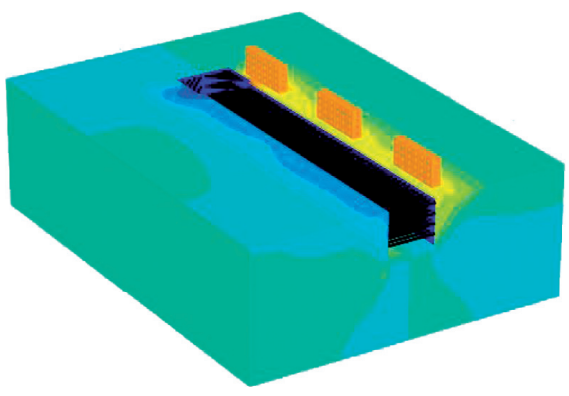

(a)

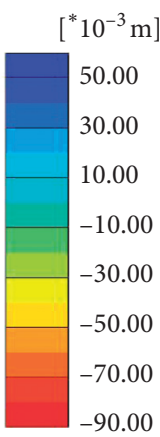

$-90.00$

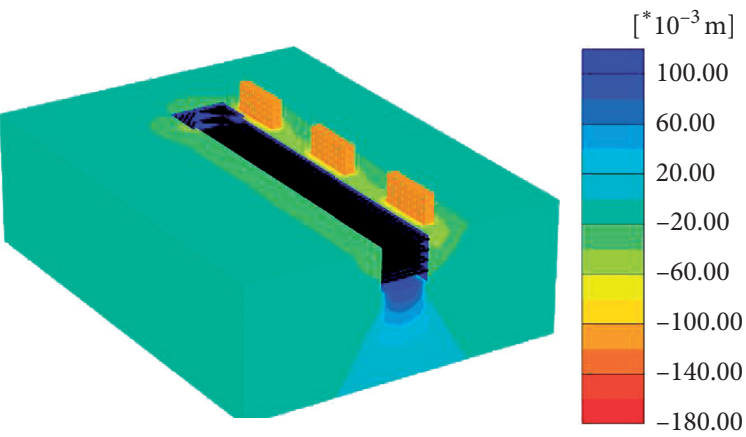

(b)

FIGURE 10: Displacement cloud image of surrounding environment after completion of foundation-pit excavation parallel to the foundation pit. (a) Horizontal displacement of soil. (b) Vertical displacement of soil.

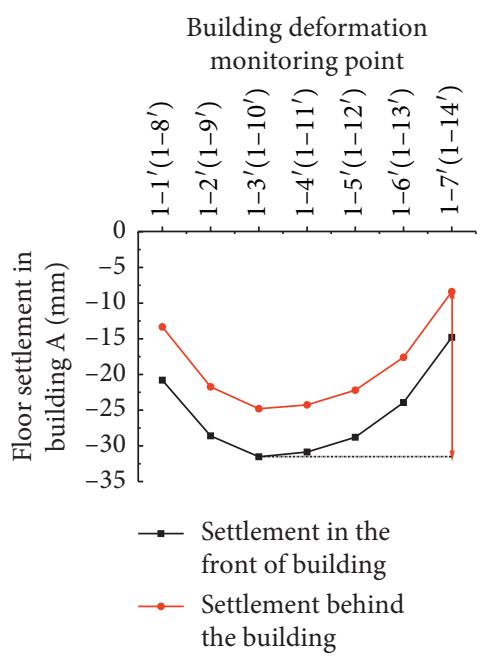

(a)

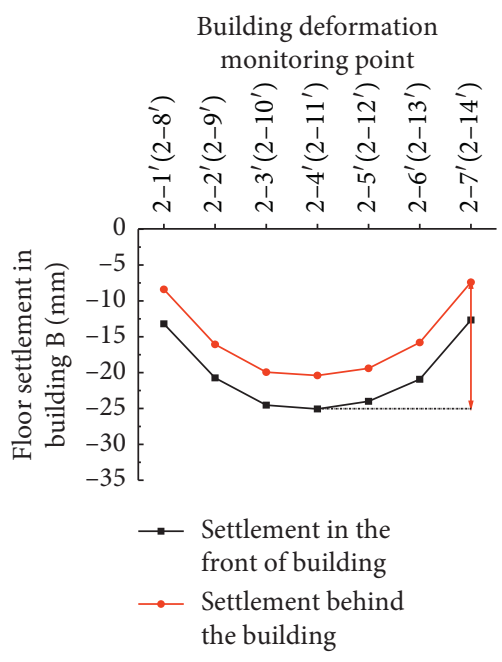

(b)

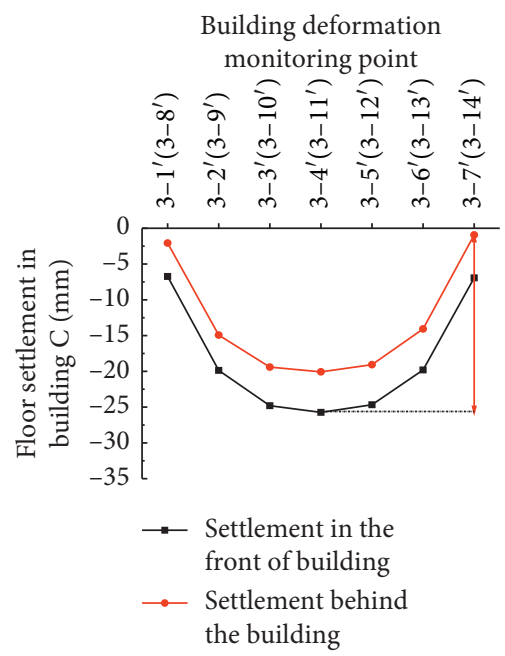

(c)

FIgUre 11: Floor settlement of the three buildings when the longitudinal wall of the building is parallel to the foundation pit. (a) Building A. (b) Building B. (c) Building C.

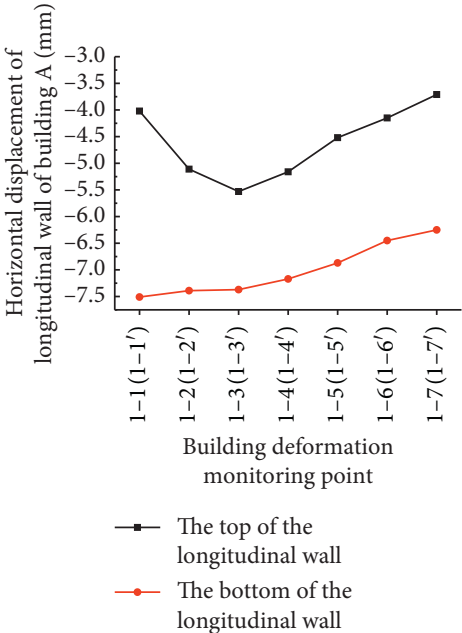

(a)

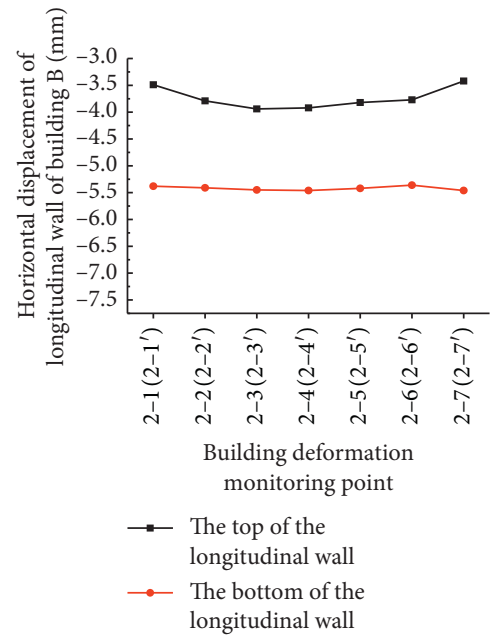

(b)

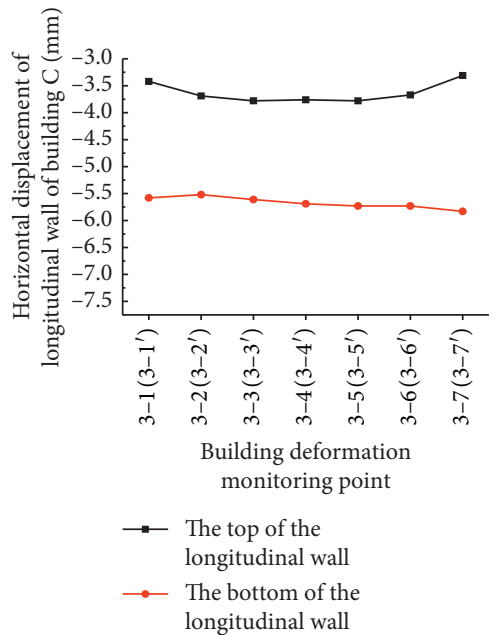

(c)

FIGURE 12: Horizontal displacement of the roof and floor of the three buildings when the longitudinal wall of the building is parallel to the foundation pit. (a) Building A. (b) Building B. (c) Building C. 


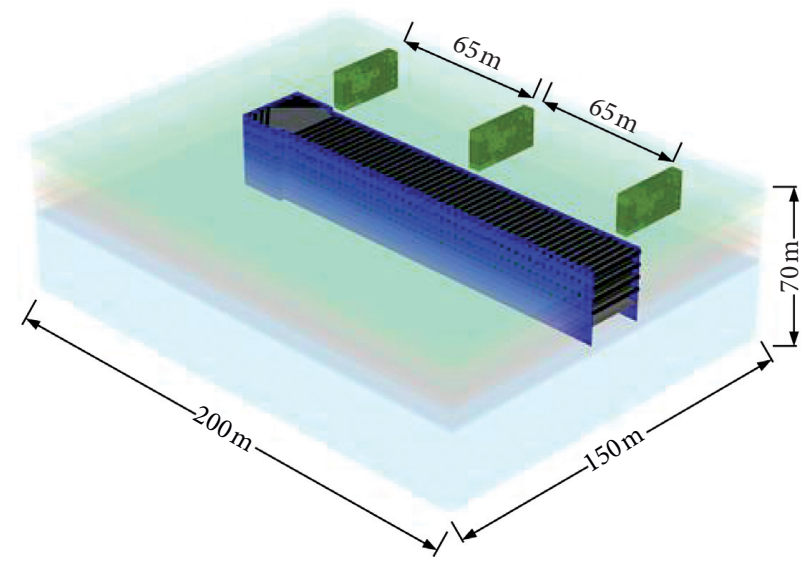

FIGURE 13: Three-dimensional numerical model of the longitudinal wall of buildings perpendicular to the foundation pit.

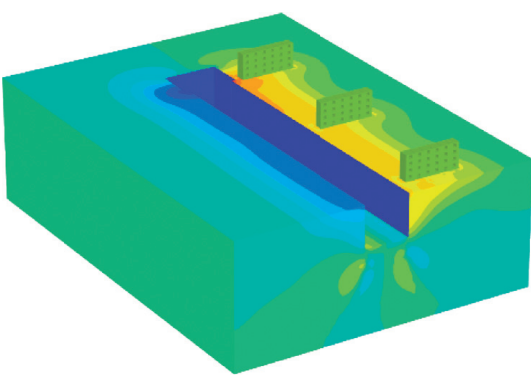

(a)

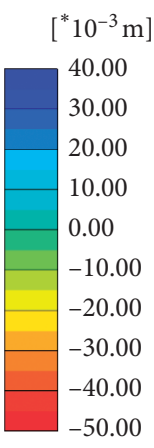

50.00

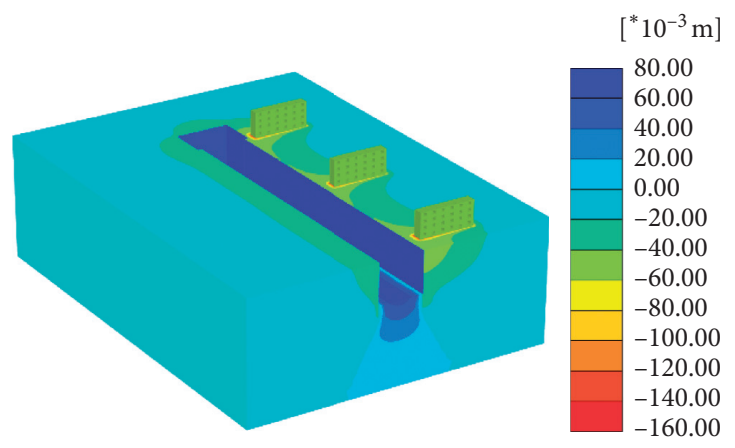

(b)

Figure 14: Displacement cloud image of surrounding environment after completion of foundation-pit excavation (perpendicular to the foundation pit). (a) Horizontal displacement of soil. (b) Vertical displacement of soil.

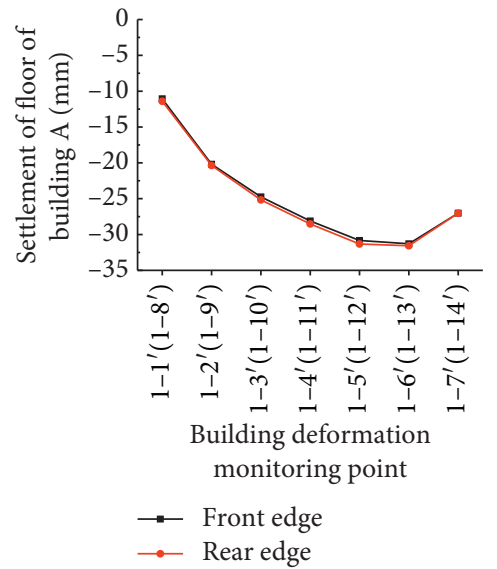

(a)

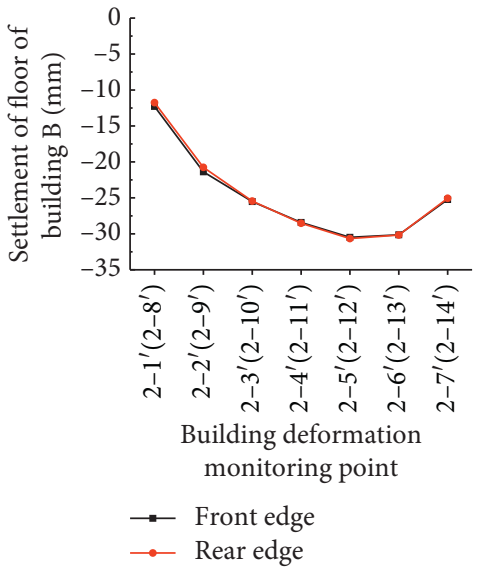

(b)

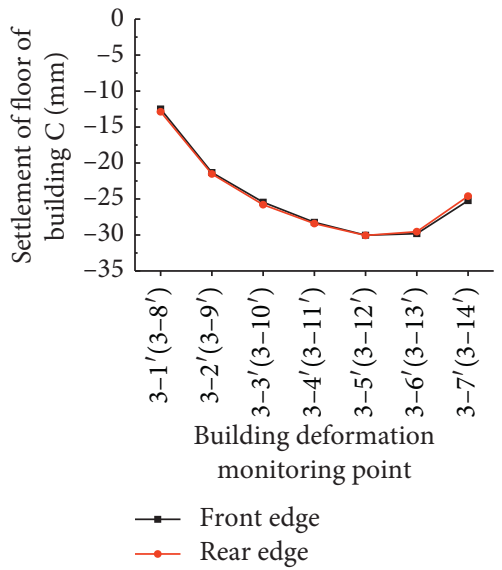

(c)

FIGURE 15: Floor settlement of the three buildings when the longitudinal wall of the building is perpendicular to the foundation pit. (a) Building A. (b) Building B. (c) Building C.

same influence factor. It is a dimensionless index and is only used to consider the degree of influence of different factors on the same deformation parameter; the larger the difference, the greater the influence of this factor on the deformation parameters. In each figure, the left curve is the degree of impact of the longitudinal walls of buildings $A, B$, 


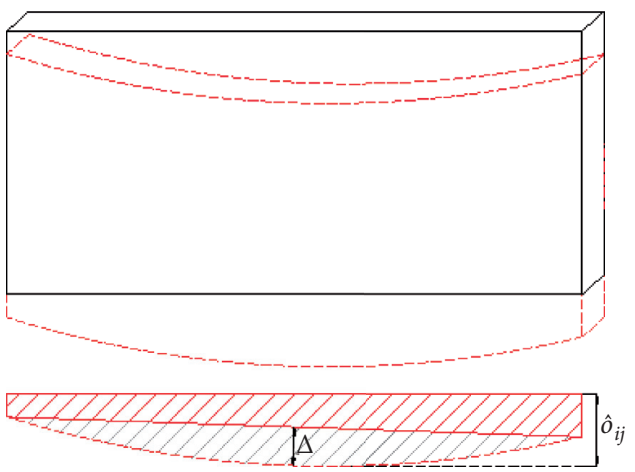

(a)

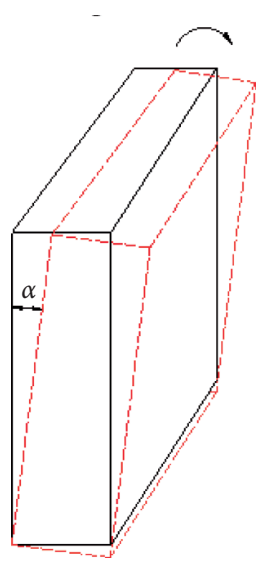

(b)

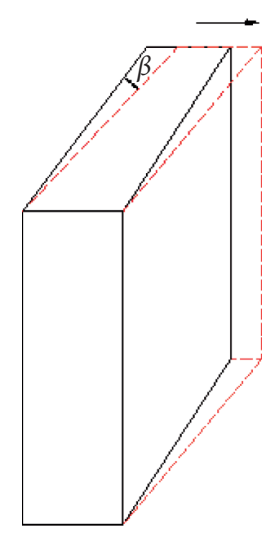

(c)

Figure 16: Diagram of building-deformation forms. (a) Relative deflection and differential settlement. (b) Tilt deformation. (c) Torsional deformation.

TABLE 5: Orthogonal test factors for a deformation response analysis of the buildings.

\begin{tabular}{lccc}
\hline \multirow{2}{*}{ Main influencing factors } & \multicolumn{3}{c}{ Test conditions } \\
& Standard 1 & Standard 2 & Standard 3 \\
\hline$s(\mathrm{~m})$ & 5 & 10 & 15 \\
$E^{c}\left(10^{3} \mathrm{MPa}\right)$ & 0.2 & 0.4 & 0.6 \\
$E^{e}\left(10^{3} \mathrm{MPa}\right)$ & 30 & 15 & 7.5 \\
$E^{s}\left(10^{3} \mathrm{MPa}\right)$ & 200 & 100 & 50 \\
\hline
\end{tabular}

and $C$ parallel to the foundation pit, and the right curve is the degree of impact of the longitudinal walls of buildings $\mathrm{A}, \mathrm{B}$, and $\mathrm{C}$ perpendicular to the foundation pit.

The comprehensive analysis set out in Figure 17 shows the following: (1) of the four influencing factors, the horizontal distance between the building and foundation pit and the stiffness of the building itself have a relatively clear influence on the deformation of the building, especially the differential settlement and relative deflection of the building; (2) regardless of whether the longitudinal wall of the building is parallel or perpendicular to the foundation pit, the degree of influence of the four deformation parameters of building $\mathrm{A}$ is larger than that of the other two locations, which is related to the complexity of the deformation near the connection between the shield shaft and the shaft section; (3) for the inclined and torsional deformation of the building in the direction of the foundation pit, in addition to the factors of the building itself, the influence of the stiffness on the bracing in the foundation pit cannot be ignored, as shown in Figures 17(a) and 17(c). The second influence is the stiffness of the foundation-pit retaining structure.

From the perspective of the relative position relationship between the building and the foundation pit, the deformation parameters of the building vary greatly with its location, and the position of building $\mathrm{A}$ is still the most clearly affected.

5.2. Interaction between Foundation-Pit Construction and Surrounding Buildings. In addition to the influence of the foundation-pit excavation on the building described above, there will also be some interaction between the building and foundation pit in the process of the foundation-pit excavation, and the degree of interaction between the two will differ because of the different design schemes of foundationpit support structures and surrounding environmental conditions. Through further analysis of the 54 testing schemes of deformation parameter trends along with the change in the main influencing factors, establishing the most and least favorable excavation and surrounding environmental conditions is possible. In this way, it is possible to not only optimize the design scheme but also design a subwaystation foundation-pit engineering and safety risk assessment targeted at taking effective control measures.

Figures 18 and 19 show building deformation under the influence of different design schemes for foundation-pit support systems and the relative position relationship, respectively. Because the differential settlement and relative deflection of the building are five orders of magnitude larger than the tilt and torsion angles, to identify the four deformation parameters simultaneously and then determine the comprehensive deformation of the building, the calculated results are normalized in the figure; i.e., the ordinate is the degree of deformation after the normalization of the four deformation parameters.

As shown in Figures 18 and 19, when the longitudinal wall of the building is parallel to the foundation pit, the scheme with the minimum comprehensive deformation of buildings $\mathrm{A}, \mathrm{B}$, and $\mathrm{C}$ is no. 3, no. 2, and no. 3, respectively, the distance between the building and foundation pit is at the minimum, stiffness of the building is medium or above, and stiffness of the retaining structure and inner support is medium or below. The maximum comprehensive deformation is no. 7 , no. 8 , and no. 7 , respectively. At this point, the distance between the building and foundation pit is the largest, building stiffness is medium or below, stiffness of the envelope structure is at the maximum or minimum, and stiffness of the inner support is medium or below.

When the longitudinal wall of the building is perpendicular to the foundation pit, the minimum comprehensive deformations of buildings $\mathrm{A}, \mathrm{B}$, and $\mathrm{C}$ are no. 8 , no. 3 , and 


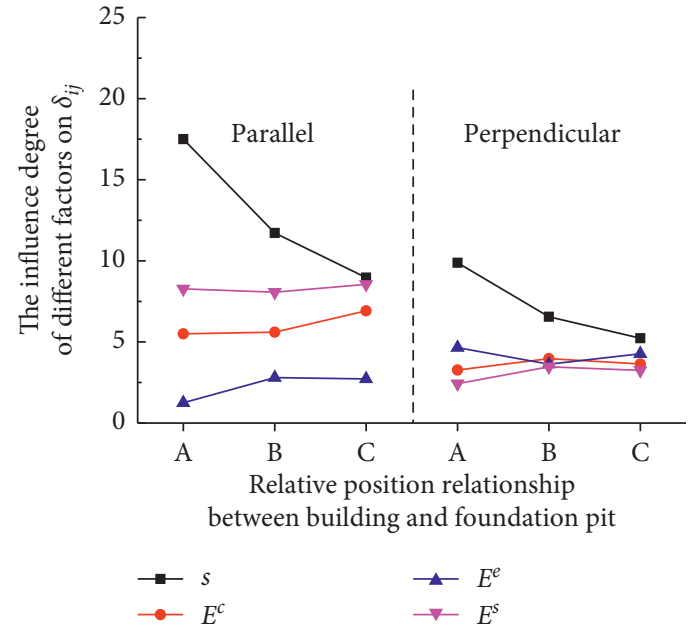

(a)

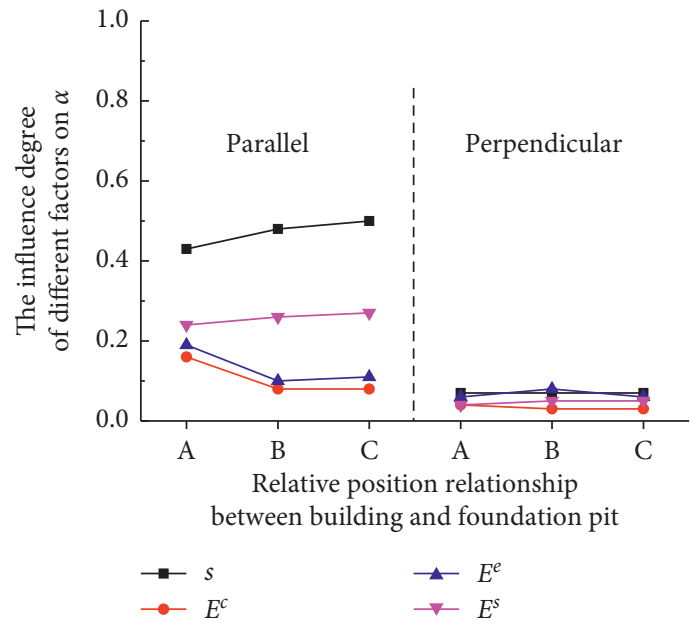

(c)

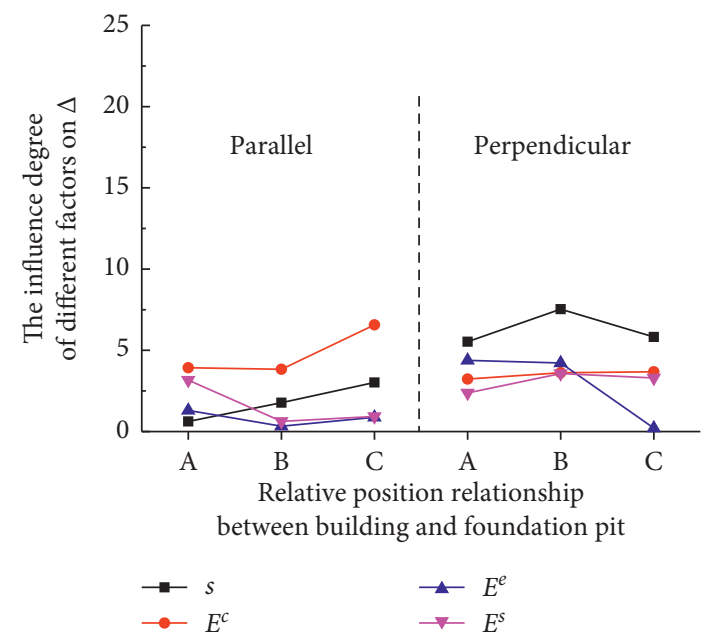

(b)

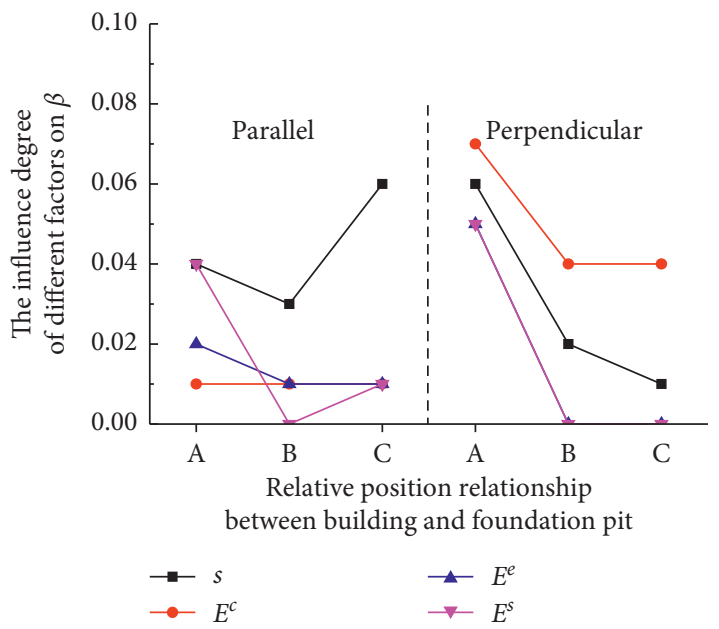

(d)

FIGURE 17: Influence of different factors and relative position on building deformation. (a) $\delta_{i j}$. (b) $\Delta$. (c) $\alpha$. (d) $\beta$.

no. 8, respectively. At this point, the distance between the building and foundation pit is at the maximum and minimum, stiffness of the building is medium and above, stiffness of the retaining structure of the foundation pit is at the maximum and minimum, and stiffness of the inner support is medium and below. The maximum comprehensive deformations are no. 4 , no. 7 , and no. 7 . In this case, the distance between the building and foundation pit is medium or above, building stiffness is at the minimum, and stiffness of the retaining structure and inner support of the foundation pit is medium or below.

Through the above analysis, it can be seen that the maximum or minimum comprehensive deformation of buildings with different relative position relationships is determined through various influencing factors. When the distance between the building and foundation pit is small, the deformation of the building may be smaller than when the distance is large. The deformation of a building with greater stiffness may also be greater than that of a building with less stiffness. Due to the diversity in forms of building deformation and influencing factors, judging the impact of foundation-pit excavation on buildings using only a single deformation or factor may lead to incomplete results. Therefore, a comprehensive evaluation method that can integrate a variety of influencing factors and deformation forms is needed.

5.3. Comprehensive Evaluation of the Deformation Response of Surrounding Buildings. This section continues the corresponding systematic evaluation of building deformation as a result of foundation-pit excavation from two perspectives: the influence of various deformation indexes and influencing factors on comprehensive deformation and the interaction between foundation-pit excavation and buildings.

5.3.1. Influence of Various Deformation Indexes on the Comprehensive Deformation of Buildings. By calculating the average value of the proportion of normalized deformation parameters in the comprehensive deformation index in the 54 schemes, the degree of influence of the four kinds of deformation on the comprehensive deformation index of the 


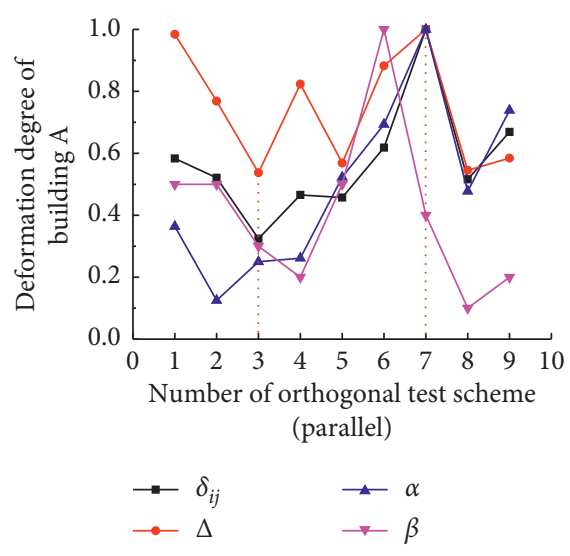

(a)

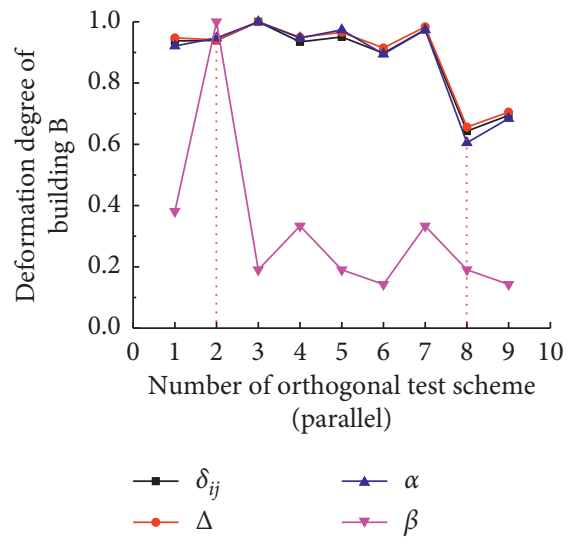

(b)

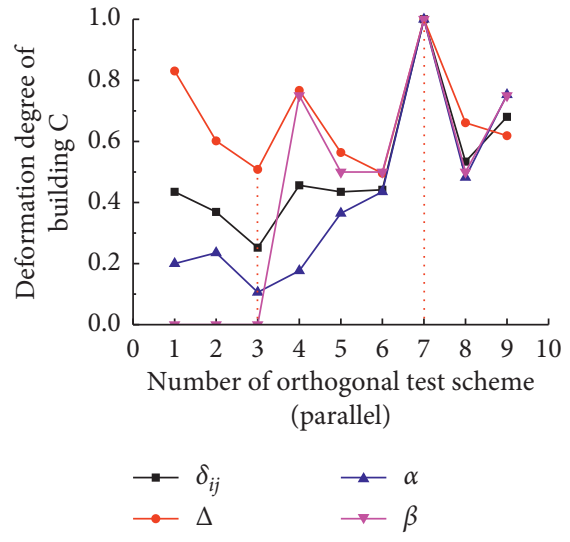

(c)

Figure 18: The variation trend of four deformation parameters in different test schemes (parallel to the foundation pit). (a) Building A. (b) Building B. (c) Building C.

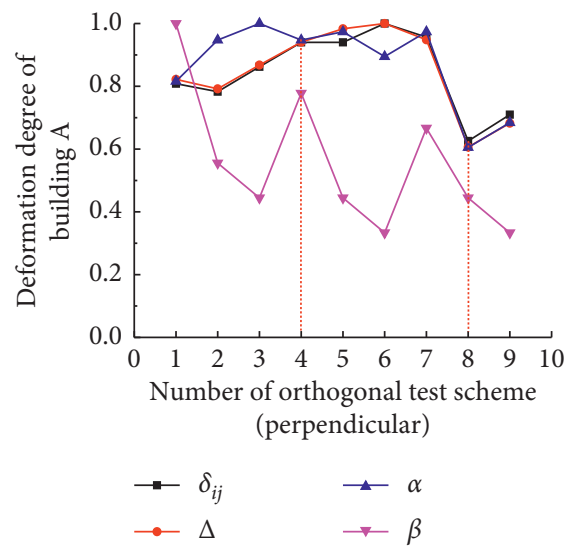

(a)

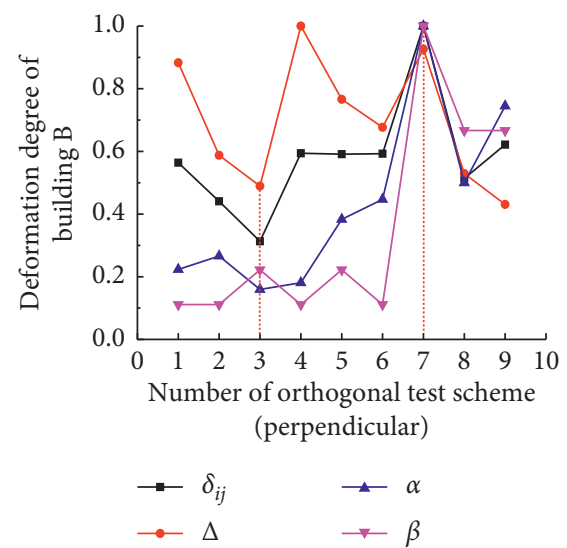

(b)

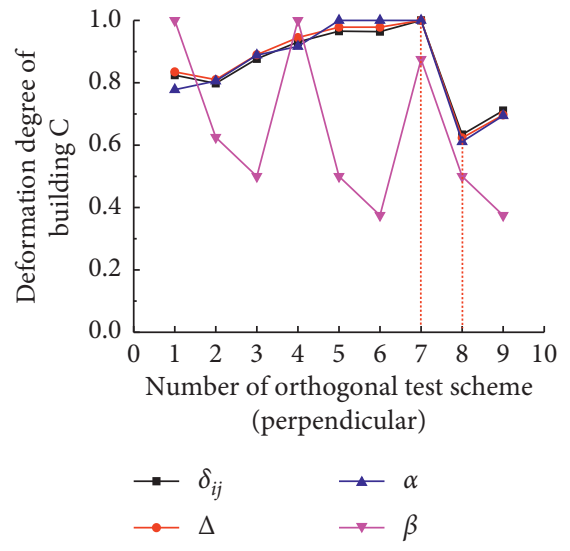

(c)

Figure 19: The variation trend of four deformation parameters in different test schemes (perpendicular to the foundation pit). (a) Building A. (b) Building B. (c) Building C.

building under different relative position relationships can be obtained, as shown in Figure 20. When the longitudinal wall of the building is parallel to the foundation pit, the relative deflection of the building has the greatest influence on the comprehensive deformation, followed by the differential settlement deformation. When the longitudinal wall of the building is perpendicular to the foundation pit, differential settlement, relative deflection, and inclined angle have an almost equal effect on the comprehensive deformation, and the torsion angle has the least effect.

In this paper, the sum of the normalized values of the four deformation indexes of the building is called the comprehensive deformation index $D_{j}$, which is used to measure the overall degree of deformation of the surrounding buildings after foundation-pit excavation. Figure 21 shows the distribution range and proportion of the comprehensive deformation index of all buildings in the 54 schemes under different relative position relationships between the buildings and foundation pits. When the longitudinal wall of the building is parallel to the foundation pit, the ratio of the comprehensive deformation index of the surrounding buildings between 1 and 3 is the largest; of these, the comprehensive deformation index between 1 and 2 accounted for $51.8 \%$, and that between 2 and 3 accounted for $29.6 \%$. The comprehensive deformation index of the building between 0 and 1 is less than 5\%, and that of more than 3 accounts for $14.8 \%$. When the longitudinal wall of the building is perpendicular to the foundation pit, the distribution of the building comprehensive deformation index between 0 and 2 is approximately zero, and the proportion between 2 and 3 accounts for $25.9 \%$; the proportion greater than 3 is the largest, accounting for $74.1 \%$.

When Figures 20 and 21 are combined, the comprehensive influence of foundation-pit excavation on the deformation of surrounding buildings under different relative position relationships can be accurately judged, as can the degree of influence of the various deformation indexes on the comprehensive deformation of the surrounding 


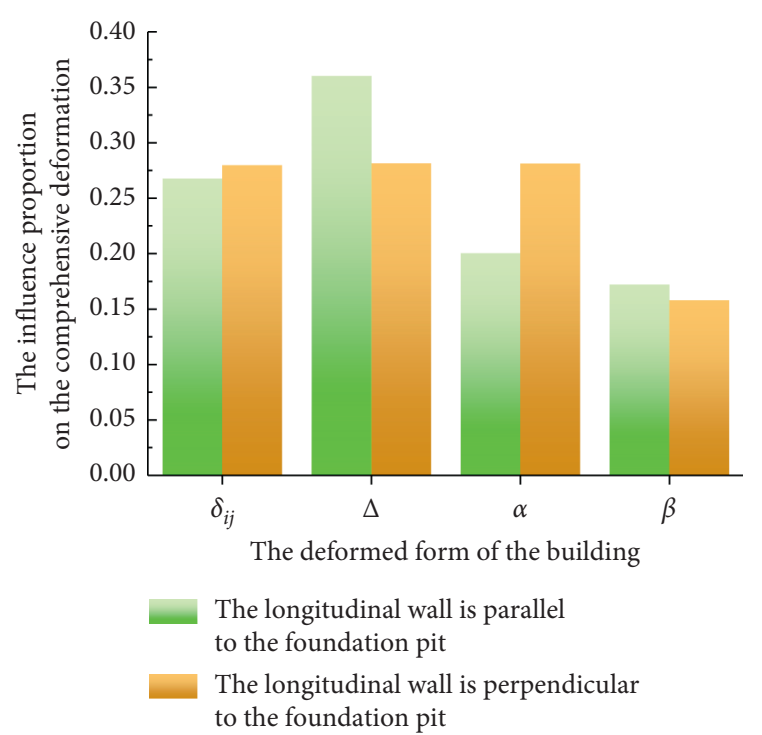

FIgURE 20: Influence of building-deformation parameters on the comprehensive deformation index.

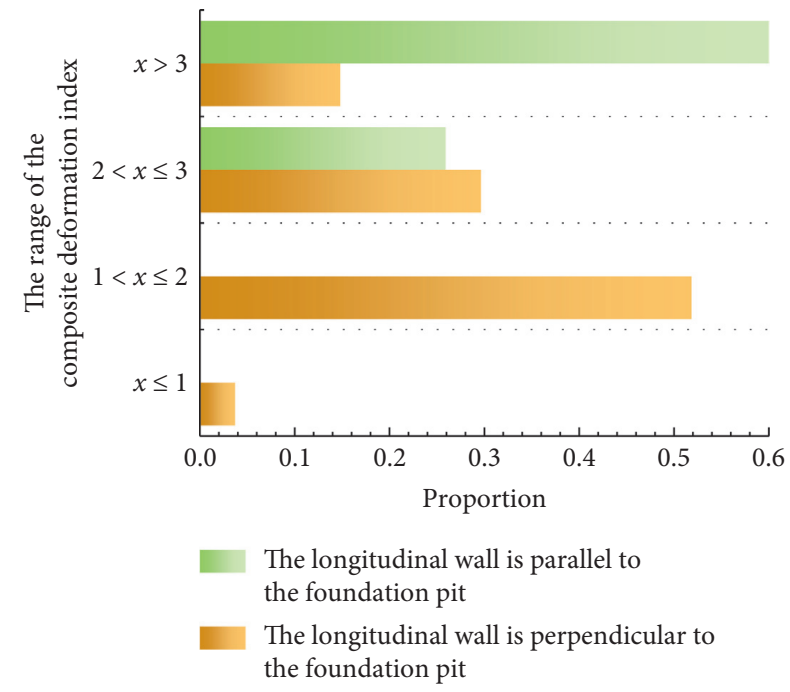

FIgURE 21: The distribution of the composite deformation index under different relative position relationships of buildings.

environment. To a certain extent, this can provide the basis for deformation prediction and the safety evaluation of similar projects.

\subsubsection{The Influence of Various Factors from the Surrounding} Environment on Comprehensive Deformation. To express the degree of influence of the four factors more intuitively, the degree of each influence factor was assigned to I, II, and III according to the order of the calculation parameters from small to large, and specific values were assigned to each level, as shown in Table 6. The average value of each deformation parameter was then calculated according to the calculation results of the 54 experimental schemes using three grades: low, medium, and high. Finally, the absolute value of the difference between the maximum and minimum values of the three average values was taken as the criterion to judge the degree of influence of the factor on each deformation parameter.

Taking differential settlement, one of the deformation parameters, as an example, in Table 7 , $\bar{a}_{i}\left(\delta^{v}\right), \bar{b}_{i}\left(\delta^{v}\right), \bar{c}_{i}\left(\delta^{v}\right)$, and $\bar{d}_{i}\left(\delta^{v}\right)$ represent the average values of the differential settlement calculated at each level $(I=1,2,3)$ of the four influencing factors, i.e., the distance between the building and foundation pit $S_{j}$, building stiffness $E_{j}^{c}$, stiffness of the retaining structure of the foundation pit $E_{j}^{e}$, and stiffness of the internal support $E_{j}^{s}$. The values $r_{s}\left(\delta^{v}\right), r_{E c}\left(\delta^{v}\right), r_{E e}\left(\delta^{v}\right)$, and $r_{E S}\left(\delta^{v}\right)$ represent the degree of influence of the four influencing factors on the differential settlement, respectively: the distance between the building and the foundation pit $S_{j}$, stiffness of the building $E_{j}^{c}$, stiffness of the retaining structure of the foundation pit $E_{j}^{e}$, and stiffness of the internal support $E_{j}^{s}$. The calculation method considers the influence of the distance between the building and foundation pit on differential settlement as an example, as shown in equations (1) and (2). The calculation method for the degree of influence of each factor on other deformation parameters can be referred to and will not be described here.

$$
\begin{aligned}
& \bar{a}_{i}\left(\delta^{\nu}\right)=\frac{\sum_{i=1}^{3} s_{i}\left(\delta^{v}\right)}{3}, \\
& r_{s}\left(\delta^{v}\right)=\operatorname{Max}\left\{\bar{a}_{i}\left(\delta^{\nu}\right)\right\}-\operatorname{Min}\left\{\bar{a}_{i}\left(\delta^{\nu}\right)\right\} .
\end{aligned}
$$

After the degree of influence of each factor on the different deformation parameters is obtained, the average value of the degree of influence of this factor on all deformation parameters is calculated, and the comprehensive influence index $R$ of each factor on building deformation is obtained. For example, the comprehensive influence index of the distance between the building and foundation pit on building deformation is shown in equation:

$$
R_{s}=\frac{r_{s}\left(\delta^{v}\right)+r_{s}(\Delta)+r_{s}(\alpha)+r_{s}(\beta)}{4},
$$

where $r_{s}(\Delta), r_{s}(\alpha)$, and $r_{s}(\beta)$ are the degree of influence of the distance between the building and foundation pit on the relative deflection, tilt angle, and torsion angle of the building, respectively. The calculation method for the comprehensive degree of influence of other factors on building deformation can be conducted using references and will not be repeated herein. Thus, the comprehensive degree of influence of the various factors on building deformation when the longitudinal wall of the building is parallel and perpendicular to the foundation pit is obtained, as shown in Table 8.

When the assigned value of each influencing factor in Table 6 is multiplied by the comprehensive degree of influence of each influencing factor in Table 8, the safety evaluation index $S_{j}$ of each scheme can be obtained. To verify the reliability of the safety evaluation index, the comprehensive deformation of the 54 schemes was sorted, as shown in Figure 22. The comprehensive deformation index is the sum of the normalized values of the deformation parameters 
TABLE 6: The grade division and assignment of influencing factors.

\begin{tabular}{lccccc}
\hline Influencing factor level & $s(\mathrm{~m})$ & $E^{c} 10^{3}(\mathrm{MPa})^{c}$ & $E^{e} 10^{3}(\mathrm{MPa})^{c}$ & $E^{s} 10^{3}(\mathrm{MPa})^{c}$ & Graphics and assignment \\
\hline I (low) & 5 & 0.2 & 7.5 & 50 & 1 \\
II (medium) & 10 & 0.4 & 15 & 100 & 2 \\
III (high) & 15 & 0.6 & 30 & 200 & 3 \\
\hline
\end{tabular}

TABle 7: Calculation table of the degree of influence of the four factors on $\delta_{i j}$.

\begin{tabular}{|c|c|c|c|c|}
\hline \multirow{2}{*}{ Level $i$} & \multicolumn{4}{|c|}{$\delta_{i j}$} \\
\hline & $s$ & $E^{c}$ & $E^{e}$ & $E^{s}$ \\
\hline 1 & $\bar{a}_{1}\left(\delta^{v}\right)$ & $\bar{b}_{1}\left(\delta^{v}\right)$ & $\bar{c}_{1}\left(\delta^{v}\right)$ & $\bar{d}_{1}\left(\delta^{v}\right)$ \\
\hline 2 & $\bar{a}_{2}\left(\delta^{v}\right)$ & $\bar{b}_{2}\left(\delta^{v}\right)$ & $\bar{c}_{2}\left(\delta^{v}\right)$ & $\bar{d}_{2}\left(\delta^{v}\right)$ \\
\hline 3 & $\bar{a}_{3}\left(\delta^{v}\right)$ & $\bar{b}_{3}\left(\delta^{v}\right)$ & $\bar{c}_{3}\left(\delta^{v}\right)$ & $\bar{d}_{3}\left(\delta^{v}\right)$ \\
\hline$r\left(\delta^{v}\right)$ & $r_{s}\left(\delta^{v}\right)$ & $r_{E c}\left(\delta^{v}\right)$ & $r_{E e}\left(\delta^{v}\right)$ & $r_{E s}\left(\delta^{v}\right)$ \\
\hline
\end{tabular}

TABle 8: The comprehensive degree of influence of various factors on building deformation.

\begin{tabular}{lccrr}
\hline Relative position relation & $s$ & $E^{c}$ & $E^{e}$ & $E^{s}$ \\
\hline Parallel to the foundation pit & 0.40 & 0.25 & 0.12 & 0.23 \\
Perpendicular to the foundation pit & 0.34 & 0.30 & 0.20 & 0.17 \\
\hline
\end{tabular}

\begin{tabular}{|c|c|c|c|c|c|c|}
\hline$L_{j}$ & $s$ & $E^{c}$ & $E^{e}$ & $E^{s}$ & $D_{j}$ & $S_{j}$ \\
\hline 9 & 3 & 3 & 2 & 3 & 1.41 & 2.91 \\
\hline 8 & 3 & 2 & 3 & 1 & 1.64 & 2.34 \\
\hline 7 & 2 & 3 & 3 & 2 & 1.75 & 2.39 \\
\hline 6 & 2 & 2 & 1 & 3 & 1.91 & 2.12 \\
\hline 5 & 2 & 1 & 2 & 1 & 2.05 & 1.55 \\
\hline 4 & 1 & 1 & 3 & 3 & 2.19 & 1.69 \\
\hline 3 & 3 & 1 & 1 & 2 & 2.43 & 2.07 \\
\hline 2 & 1 & 2 & 2 & 2 & 3.19 & 1.6 \\
\hline 1 & 1 & 3 & 1 & 1 & 3.40 & 1.51 \\
\hline
\end{tabular}

\begin{tabular}{|c|c|c|c|c|c|c|}
\hline$L_{j}$ & $s$ & $E^{c}$ & $E^{e}$ & $E^{s}$ & $D_{j}$ & $S_{j}$ \\
\hline 9 & 3 & 2 & 3 & 1 & 2.10 & 2.36 \\
\hline 8 & 3 & 3 & 2 & 3 & 2.23 & 2.78 \\
\hline 7 & 1 & 2 & 2 & 2 & 2.85 & 1.63 \\
\hline 6 & 1 & 3 & 1 & 1 & 3.08 & 1.57 \\
\hline 5 & 2 & 3 & 3 & 2 & 3.16 & 2.46 \\
\hline 4 & 2 & 2 & 1 & 3 & 3.19 & 1.95 \\
\hline 3 & 1 & 1 & 3 & 3 & 3.19 & 1.69 \\
\hline 2 & 3 & 1 & 1 & 2 & 3.27 & 1.85 \\
\hline 1 & 2 & 1 & 2 & 1 & 3.83 & 1.53 \\
\hline
\end{tabular}

(a)

\begin{tabular}{|c|c|c|c|c|c|c|}
\hline$L_{j}$ & $s$ & $E^{c}$ & $E^{e}$ & $E^{s}$ & $D_{j}$ & $S_{j}$ \\
\hline 9 & 3 & 3 & 2 & 3 & 0.87 & 2.91 \\
\hline 8 & 2 & 3 & 3 & 2 & 1.21 & 2.39 \\
\hline 7 & 3 & 2 & 3 & 1 & 1.47 & 2.34 \\
\hline 6 & 3 & 1 & 1 & 2 & 1.86 & 2.07 \\
\hline 5 & 2 & 2 & 1 & 3 & 1.87 & 2.12 \\
\hline 4 & 1 & 2 & 2 & 2 & 2.15 & 1.6 \\
\hline 3 & 1 & 1 & 3 & 3 & 2.18 & 1.69 \\
\hline 2 & 2 & 1 & 2 & 1 & 2.80 & 1.55 \\
\hline 1 & 1 & 3 & 1 & 1 & 4.0 & 1.51 \\
\hline
\end{tabular}

\begin{tabular}{|c|c|c|c|c|c|c|}
\hline$L_{j}$ & $s$ & $E^{c}$ & $E^{e}$ & $E^{s}$ & $D_{j}$ & $S_{j}$ \\
\hline 9 & 3 & 3 & 2 & 3 & 2.28 & 2.78 \\
\hline 8 & 2 & 3 & 3 & 2 & 2.41 & 2.46 \\
\hline 7 & 3 & 2 & 3 & 1 & 3.08 & 2.36 \\
\hline 6 & 2 & 2 & 1 & 3 & 3.17 & 1.95 \\
\hline 5 & 3 & 1 & 1 & 2 & 3.23 & 1.85 \\
\hline 4 & 1 & 1 & 3 & 3 & 3.34 & 1.69 \\
\hline 3 & 1 & 2 & 2 & 2 & 3.45 & 1.63 \\
\hline 2 & 2 & 1 & 2 & 1 & 3.55 & 1.53 \\
\hline 1 & 1 & 3 & 1 & 1 & 3.61 & 1.57 \\
\hline
\end{tabular}

(b)

FIgURE 22: Continued. 


\begin{tabular}{|c|c|c|c|c|c|c|}
\hline$L_{j}$ & $s$ & $E^{c}$ & $E^{e}$ & $E^{s}$ & $D_{j}$ & $S_{j}$ \\
\hline 9 & 3 & 3 & 2 & 3 & 1.18 & 2.91 \\
\hline 8 & 3 & 2 & 3 & 1 & 1.41 & 2.34 \\
\hline 7 & 2 & 3 & 3 & 2 & 1.78 & 2.39 \\
\hline 6 & 3 & 1 & 1 & 2 & 1.83 & 2.07 \\
\hline 5 & 2 & 2 & 1 & 3 & 1.89 & 2.12 \\
\hline 4 & 1 & 1 & 3 & 3 & 1.96 & 1.69 \\
\hline 3 & 1 & 2 & 2 & 2 & 2.21 & 1.6 \\
\hline 2 & 2 & 1 & 2 & 1 & 2.46 & 1.55 \\
\hline 1 & 1 & 3 & 1 & 1 & 3.93 & 1.51 \\
\hline
\end{tabular}

\begin{tabular}{|c|c|c|c|c|c|c|}
\hline$L_{j}$ & $s$ & $E^{c}$ & $E^{e}$ & $E^{s}$ & $D_{j}$ & $S_{j}$ \\
\hline 9 & 3 & 3 & 2 & 3 & 2.37 & 2.78 \\
\hline 8 & 2 & 3 & 3 & 2 & 2.48 & 2.46 \\
\hline 7 & 3 & 2 & 3 & 1 & 3.04 & 2.36 \\
\hline 6 & 3 & 1 & 1 & 2 & 3.16 & 1.85 \\
\hline 5 & 2 & 2 & 1 & 3 & 3.32 & 1.95 \\
\hline 4 & 1 & 1 & 3 & 3 & 3.44 & 1.69 \\
\hline 3 & 1 & 2 & 2 & 2 & 3.44 & 1.63 \\
\hline 2 & 2 & 1 & 2 & 1 & 3.79 & 1.53 \\
\hline 1 & 1 & 3 & 1 & 1 & 3.88 & 1.57 \\
\hline
\end{tabular}

(c)

FIGURE 22: Comprehensive deformation index $\left(D_{j}\right)$ and safety evaluation index $\left(S_{j}\right)$ of surrounding buildings under different schemes. (a) Building A. (b) Building B. (c) Building C.

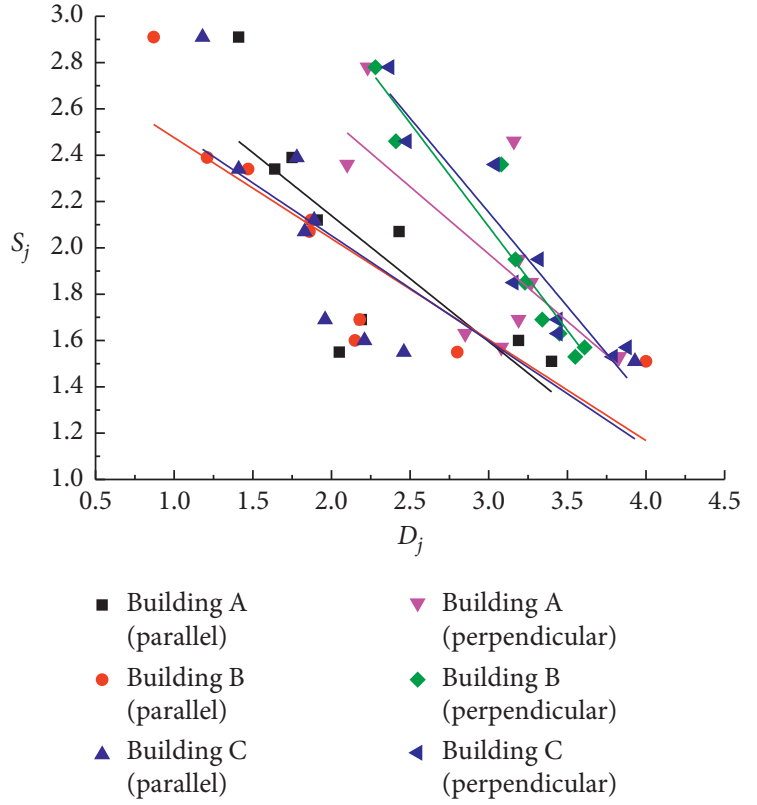

FIGURE 23: Relationship between the comprehensive deformation index $\left(D_{j}\right)$ and safety evaluation index $\left(S_{j}\right)$ of surrounding buildings.

corresponding to each scheme in Figures 18 and 19: the larger the sum, the greater the comprehensive deformation index; the smaller the sum, the lower the comprehensive deformation index; and the smaller the comprehensive deformation index, the higher the safety level of the scheme.

Therefore, the comprehensive deformation index is sorted from small to large, and the corresponding safety level of each scheme is ranked from high to low, as shown in columns 1 and 6 of each table in Figure 22. Columns 2 to 5 are the grades of the four influencing factors corresponding to each scheme and are multiplied by the comprehensive degree of influence of each influencing factor on building deformation under the corresponding relative position

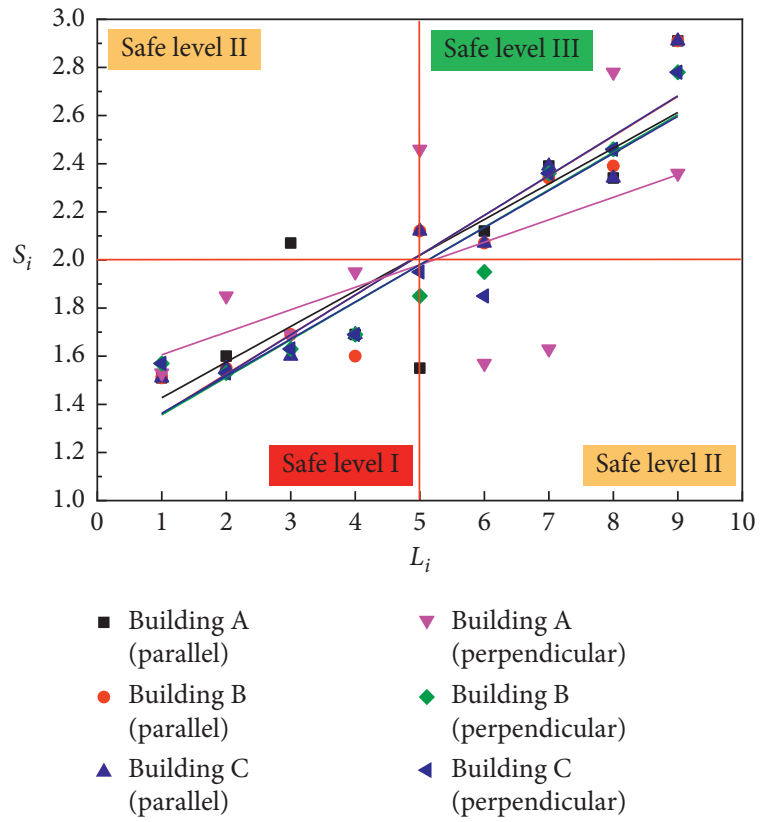

FIgURE 24: The relationship between the safety grade $\left(L_{j}\right)$ of the scheme and safety evaluation index $\left(S_{j}\right)$ of the surrounding buildings.

relationship set out in Table 8; the corresponding safety evaluation index $S_{j}$ can be obtained for each scheme.

As can be observed, with an increase in the comprehensive deformation index of each table in Figure 22, the corresponding safety evaluation index shows a downward trend. This rule can be expressed intuitively, as in Figure 23. The relationship between the building comprehensive deformation index $D_{j}$ and safety evaluation index $S_{j}$ can be expressed by (4), where $-0.89<m \leq-0.43,2.9<n \leq 4.8$ :

$$
D_{j}=m S_{j}+n .
$$

Moreover, according to Figure 22, the relationship between the scheme safety level $L_{j}$ and safety evaluation index $S_{j}$ can be obtained, as shown in Figure 24. A certain linear 
TABLE 9: Safety evaluation of building-deformation response during foundation-pit excavation and corresponding grade description.

\begin{tabular}{|c|c|c|c|c|}
\hline \multirow{2}{*}{$\begin{array}{l}\text { Safety level } \\
\text { evaluation }\end{array}$} & \multicolumn{2}{|c|}{$\begin{array}{c}\text { Basis of } \\
\text { evaluation }\end{array}$} & \multirow[t]{2}{*}{ Description of basis } & \multirow[t]{2}{*}{ Description of the safety degree } \\
\hline & $S_{j}$ & $L_{j}$ & & \\
\hline Level III & $2 \sim 3$ & $5 \sim 9$ & $\begin{array}{l}\text { Both the safety evaluation index and the } \\
\text { safety grade of the scheme are high }\end{array}$ & $\begin{array}{l}\text { The safety reserve of the foundation-pit design scheme is } \\
\text { larger or the surrounding environment deformation is less } \\
\text { affected by the excavation of the foundation pit }\end{array}$ \\
\hline \multirow[t]{2}{*}{ Level II } & $2 \sim 3$ & $1 \sim 5$ & $\begin{array}{l}\text { The safety evaluation index is higher, } \\
\text { but the safety grade of the scheme is } \\
\text { lower }\end{array}$ & $\begin{array}{l}\text { The safety reserve of the foundation-pit design scheme is small } \\
\text { or the surrounding environment deformation is less affected } \\
\text { by the foundation-pit excavation }\end{array}$ \\
\hline & $1 \sim 2$ & $5 \sim 9$ & $\begin{array}{l}\text { The safety level of the scheme is higher, } \\
\text { but the safety evaluatioxn index is lower }\end{array}$ & $\begin{array}{l}\text { The design of the foundation pit has a high safety reserve or } \\
\text { the surrounding environment or the deformation of the } \\
\text { surrounding environment is greatly affected by the excavation } \\
\text { of the foundation pit }\end{array}$ \\
\hline Level I & $1 \sim 2$ & $1 \sim 5$ & $\begin{array}{l}\text { Both the safety evaluation index and the } \\
\text { safety grade of the scheme are lower }\end{array}$ & $\begin{array}{l}\text { The safety reserve of the foundation-pit design is lower or the } \\
\text { surrounding environment or the deformation of the } \\
\text { surrounding environment is greatly affected by the excavation } \\
\text { of the foundation pit }\end{array}$ \\
\hline
\end{tabular}

relationship exists between the two, as shown in equation (5), where $0.09<a \leq 0.17,1.2<b \leq 1.5$ :

$$
S_{j}=a L_{j}+b
$$

Figure 24 can be further divided to clarify the criterion for the environmental impact of the relationship between the environmental safety evaluation index around foundation pit and the safety level of the scheme. In this figure, the relationship between the safety evaluation index of the building and safety level of the scheme has a common intersection point, which is roughly located at the positions of $S_{j}=2$ and $L_{j}=5$. With this intersection point as the center, the horizontal and vertical symmetrical middle lines and red line segments in Figure 24 can be drawn. The two lines divide the diagram into four parts so that the environmental safety situation can be judged simultaneously according to the safety evaluation index and safety level of the scheme, as shown in Table 9.

\section{Conclusion}

(1) The horizontal distance between the building and foundation pit and stiffness of the building itself are the factors that have a clear influence on the deformation of the building, especially the difference settlement and relative deflection of the building. Regardless of whether the longitudinal wall of the building is perpendicular or parallel to the foundation pit, the influence of the four deformation parameters of the building near the pit angle is greater than that of the building at the other two locations. For the tilt and torsion of the building toward the foundation pit, in addition to the factors relating to the building itself, the influence of the stiffness of the bracing in the foundation pit cannot be ignored.

(2) When the longitudinal wall of the building is parallel to the foundation pit, the relative deflection of the building has the greatest influence on the comprehensive deformation, followed by the differential settlement deformation. The proportion of distribution of the building comprehensive deformation index is the largest between 1 and 3. When the longitudinal wall of the building is perpendicular to the foundation pit, the differential settlement, relative deflection, and inclined-angle deformation of the building have the same effect on the comprehensive deformation, and the torsion angle has the least effect; the proportion of the comprehensive deformation index greater than 3 is the largest.

(3) The influence of various factors relating to the surrounding environment on the comprehensive deformation can be determined by defining the comprehensive deformation index $D_{j}$, peripheral environmental safety evaluation index $S_{j}$, and scheme safety levels $L_{j}$; then further analysis of the relationship among the three indexes is based on the above three indicators. By combining the comprehensive deformation arrangement scheme safety grade with the degree of influence of the various factors on comprehensive deformation, an environmental response evaluation method of the multiangle safety evaluation index can be obtained.

\section{Glossary}

$L$ : Length of the foundation pit's retaining structure

$B$ : Width of the foundation pit's retaining structure

$H_{\mathrm{e}}$ : Excavation depth of the foundation pit's retaining structure

$H$ : Depth of the foundation pit's retaining structure

$d$ : Diameter of the bored pile

$l$ : Spacing of the bored pile

$H$ : Depth of the bored pile

$E$ : Stiffness of the bored pile

$\delta_{i j}$ : Primarily uneven settlement

$\Delta:$ Overall deflection

$\alpha$ : Inclination toward the pit

$\beta$ : Torsion 
$s$ : The horizontal distance between the building and foundation pit

$E_{c}$ : The stiffness of the building wall

$E^{e}$ : The stiffness of the retaining structure

$E^{s}$ : The stiffness of the internal support

$D_{j}$ : The comprehensive deformation index

$R$ : The comprehensive influence index

$S_{j}$ : The corresponding safety evaluation index

$L_{j}$ : The scheme safety level.

\section{Data Availability}

The measured data used to support the findings of this study are included within the article.

\section{Conflicts of Interest}

The authors declare that they have no conflicts of interest.

\section{Acknowledgments}

This research was supported by the National Natural Science Foundation of China, Grant no. 51278233, and Science and Technology Project of China Communications First Bureau Group Co. Ltd, Grant no. 4GS(J)-GUD-GCL-01-JS-016.

\section{References}

[1] H. Bin, X. Li, and L. Zheng, "Influences of urban viaduct pier caps on uneven settlement of roadbed," Advances in Civil Engineering, vol. 2019, Article ID 4151278, 20 pages, 2019.

[2] H. Li, E. Ma, J. Lai et al., "Tunnelling-Induced settlement and treatment techniques for a loess metro in Xi'an," Advances in Civil Engineering, vol. 2020, Article ID 1854813, 20 pages, 2020.

[3] X. Weng, R. Zhou, W. Rao, and D. Wang, "Research on subway shield tunnel induced by local water immersion of collapsible loess," Natural Hazards, vol. 2021, 2021.

[4] Q. Fang, J. Du, J. Li, D. Zhang, and L. Cao, "Settlement characteristics of large-diameter shield excavation below existing subway in close vicinity," Journal of Central South University, vol. 28, no. 3, pp. 882-897, 2021.

[5] H. Zheng, P. Li, and G. Ma, "Stability analysis of the middle soil pillar for asymmetric parallel tunnels by using model testing and numerical simulations," Tunnelling and Underground Space Technology, vol. 108, Article ID 103686, 2020.

[6] S. He, J. Lai, Y. Zhong et al., "Damage behaviors, prediction methods and prevention methods of rockburst in 13 deep traffic tunnels in China," Engineering Failure Analysis, vol. 121, Article ID 105178, 2021.

[7] S. Xu, H. Lei, C. Li, H. Liu, J. Lai, and T. Liu, "Model test on mechanical characteristics of shallow tunnel excavation failure in gully topography," Engineering Failure Analysis, vol. 119, Article ID 104978, 2021.

[8] D. Li and C. Yan, "Building deformation prediction based on ground surface settlements of metro-station deep excavation," Advances in Civil Engineering, vol. 2018, Article ID 6050353, 14 pages, 2018.

[9] Y.-Y. Liang, N.-W. Liu, F. Yu, X.-N. Gong, and Y.-T. Chen, "Prediction of response of existing building piles to adjacent deep excavation in soft clay," Advances in Civil Engineering, vol. 2019, Article ID 8914708, 11 pages, 2019.
[10] J. Zhang, R. Zhang, L. Ding, Q. Chen, and D. Zhao, "Displacement control effects and parameter analysis of passive zone improvement of foundation pits," Chinese Journal of Rock Mechanics and Engineering, vol. 29, no. 5, pp. 1042-1051, 2010.

[11] Q. Cheng, G. Liu, and W. Zhang, "Deformation analysis of subway ultra-deep and deep excavation in Shanghai by FEM," Chinese Journal of Under-ground Space and Engineering, vol. 5, no. 2, pp. 1497-1502, 2009.

[12] Z. Li, W. Lin, Y. Zhu et al., "Field test and analysis of controlling metro excavations deformation by foundation reinforcement," Journal of Zhejiang University (Engineering Edition), vol. 51, no. 8, pp. 1475-1481, 2017.

[13] X. Ren, Analysis of the Effect of Deformation on the Shanghai Subway Station Foundation Pit Basement Reinforcement, Tongji University, Shanghai, China, 2009.

[14] E. J. Cording, T. D. O’Rourke, and M. Boscardin, "Ground movements and damage to structures," in Proceedings of the International Conference on Evaluation and Prediction of Subsidence, S. K. Saxena, Ed., ASCE, New York, NY, USA, pp. 516-537, November 1978.

[15] S. J. Boone, J. Westland, and R. Nusink, "Comparative evaluation of building responses to an adjacent braced excavation," Canadian Geotechnical Journal, vol. 36, no. 2, pp. 210-223, 1999.

[16] A. W. Skempton and D. H. MacDonald, "The allowable settlements of buildings," Proceedings of the Institution of Civil Engineers, vol. 5, no. 6, pp. 727-768, 1956.

[17] J. B. Burland, "Assessment of risk of damage to buildings due to tunneling and excavations. Invited special lecture," in Proceedings of the First International Conference on Earthquake Geotechnical Engineering, IS-Tokyo'95, Tokyo, Japan, November 1995.

[18] J. B. Burland and C. P. Wroth, "Settlement of buildings and associated damage," in Proceedings of the Conference on Settlement of Structures, pp. 611-654, Pentech Press, Cambridge, UK, January 1974.

[19] S. Li, D. Zhang, Q. Fang, and W. Lu, "Research on characteristics of ground surface deformation during deep excavation in Beijing subway," Chinese Journal of Rock Mechanics and Engineering, vol. 31, no. 1, pp. 189-198, 2012.

[20] Z. Li and G. Zheng, "Finite element analysis of response of building with different stiffnesses adjacent to excavation," Rock and Soil Mechanics, vol. 34, no. 6, pp. 1807-1814, 2013.

[21] G. Zheng and Z. Li, "Finite element analysis of adjacent building response to corner effect of excavation," Journal of Tianjin University, vol. 45, no. 8, pp. 688-699, 2012.

[22] G. B. Liu, R. J. Jiang, C. W. W. Ng, and Y. Hong, "Deformation characteristics of a $38 \mathrm{~m}$ deep excavation in soft clay," $\mathrm{Ca}$ nadian Geotechnical Journal, vol. 48, no. 12, pp. 1817-1828, 2011.

[23] N. Liu, Y. Chen, X. Gong et al., "Analysis of deformation characteristics of foundation pit of metro station and adjacent buildings induced by deep excavation in soft soil," Rock and Soil Mechanics, vol. 40, no. 4, pp. 1515-1525, 2019.

[24] P. G. Hsieh and C.-Y. Ou, "Shape of ground surface settlement profiles caused by excavation," Canadian Geotechnical Journal, vol. 35, no. 6, pp. 1004-1017, 1998.

[25] T. Benz, Small Strain Stiffness of Soils and its Numerical consequences, Institute of Geotechnical Engineering, University of Stuttgart, Stuttgart, Germany, 2007. 